\title{
3 Notfallstufen: qualitative Unterschiede in der Notfallversorgung
}

Laura Oschmann, Ulrike Nimptsch, Martin Möckel, Claudia Römer und Alexander Geissler

\subsection{Einleitung}

Die Notfallversorgung steht vor vielfältigen Herausforderungen. Einerseits kommt es seit Jahren zu einer steigenden Anzahl von Notfällen in den Notaufnahmen, die in ihrer Behandlungsdringlichkeit stark variieren. Die Ursachen dafür sind vielfältig und reichen von mangelnden ambulanten Angeboten (Schmiedhofer et al. 2016) bis hin zu unzureichender Koordination zwischen den Sektoren (Somasundaram et al. 2018). Andererseits ist vielerorts die Abstimmung mit dem regionalen Rettungsdienst durch fehlende Kommunikationsstrukturen ausbaufähig (Geissler et al. 2017) und zusätzlich ist eine Steigerung von Akutzuweisungen (Rittberg et al. 2020) durch den Rettungsdienst zu verzeichnen. Daneben sind längst nicht alle Standorte personell und infrastrukturell entsprechend ausgestattet, um jederzeit alle Notfallarten zu versorgen. In Summe mündet dies in einer für zahlreiche Indikationen nachgewiesenen Qualitätsvariation, die im Notfall eine adäquate Versorgung gefährdet und weder für die Rettungsdienste noch für Patienten transparent ist (Nimptsch u. Mansky 2017 sowie Pross et al. 2017).

Der Gesetzgeber hat mit einer Reihe von Maßnahmen, z.B. der Schaffung von Portal-, Anlauf- oder Notdienstpraxen an Krankenhäusern oder zuletzt mit der Einführung eines gestuften Systems von Notfallstrukturen für die stationäre Notfallversorgung, auf einen Teil dieser Herausforderungen reagiert $(\$ 136 \mathrm{C}$ Abs. 4 Beschlüsse des Gemeinsamen Bundesausschusses zu Qualitätssicherung 
und Krankenhausplanung). Der Gemeinsame Bundesausschuss (G-BA) war mit der Umsetzung betraut und beschloss 2018 (Struktur-)Kriterien für jede Notfallstufe. Dabei müssen je nach Stufe konkrete personelle und technische Anforderungen erfüllt werden (G-BA 2018). Daneben ist die Notfallstufenzuordnung an eine Finanzierungszusage geknüpft, um die Krankenhäuser entsprechend ihrer Ausstattung und für die Vorhaltung von stationären Notfallkapazitäten zu unterstützen (GKV-Spitzenverband 2018). Krankenhäuser erhalten demnach jährlich, auf Grundlage der vereinbarten Notfallstufe, einen pauschalen Zuschlag.

\section{Anforderungen der Notfallstufen und Module}

Die Notfallstufe bemisst sich abhängig von den verfügbaren Fachabteilungen, dem vorhandenen Fachpersonal und der zur Verfügung stehenden technischen Ausstattung. Dabei wird ein Krankenhaus ab dem Jahr 2019 entweder der Basisnotfallversorgung (Stufe 1), der erweiterten Notfallversorgung (Stufe 2) oder der umfassenden Notfallversorgung (Stufe 3) zugeordnet. Gleichwohl gilt der Grundsatz: Sollte ein Krankenhaus die Anforderungen zur Teilnahme an der Basisnotfallversorgung nicht erfüllen, kann es durch die Teilnahme an einem der zusätzlichen festgelegten Module, z.B. in der Schlaganfallversorgung oder bei Durchblutungsstörungen am Herzen, der Basisnotfallversorgung zugeordnet werden. Daraus ergibt sich, dass der Aufwand von Krankenhäusern, die keine Notfallstufe vereinbart haben, über die Teilnahme an den speziellen Modulen vergütet werden kann.

Die zusätzliche Vergütung der Krankenhäuser für eine Teilnahme an der Notfallversorgung soll die Vorhaltung von Notfallkapazitäten über pauschalierte Zuschläge sicherstellen. Den G-BA-Regelungen über die Notfallstufen folgend ist die Fallzahl in den Notaufnahmen dabei nicht relevant. Vielmehr wird lediglich die personelle und infrastrukturelle Ausstattung bei der Zuordnung der jeweiligen Stufe berücksichtigt. Bei allen drei Stufen muss in der Zentralen Notaufnahme (ZNA) die Behandlungspriorität des eintreffenden Patienten innerhalb von 10 Minuten eingeschätzt werden. Notfallstufenübergreifend muss außerdem in den Fachabteilungen dem Patienten innerhalb von $30 \mathrm{Mi}-$ nuten ein Facharzt zur Verfügung stehen. Um der Notfallstufe 2 oder 3 zugeordnet werden zu können, ist zusätzlich die Vorhaltung weiterer vier bzw. sieben Fachabteilungen erforderlich. Unter diesen können sich optional die Fachabteilungen der Kardiologie, der Neurochirurgie und Neurologie und der Orthopädie und Unfallchirurgie befinden (neben weiteren Abteilungen wie beispielsweise der Frauenheilkunde).

Für die Teilnahme an der Notfallstufe 1 erhält ein Krankenhaus eine jährliche Pauschale von 153.00o Euro. Bei Teilnahme an der Notfallstufe 2 sind es jährlich 459.00o Euro, an der Notfallstufe 3 689.ooo Euro (Stand 2019). Krankenhäuser, die nicht an der Notfallversorgung teilnehmen, müssen einen Abschlag von 6o Euro je Fallpauschale hinnehmen. 
Wie beschrieben ist es möglich, dass ein Krankenhaus sowohl das Modul der Schlaganfallversorgung als auch der Durchblutungsstörungen am Herzen (Abs. 1 Satz 2 Nr. 5) vereinbart. Ein pauschaler Zuschlag kann in beiden Fällen nur festgelegt werden, sofern das Haus keiner der Notfallstufen zugeordnet wurde. Dieser Zuschlag wird daran bemessen, wie hoch der prozentuale Anteil der vollstationären Fälle in der Stroke Unit und/oder der Chest Pain Unit am gesamten Fallaufkommen vollstationärer Fälle des Krankenhauses im vorangegangenen Jahr gewesen ist (GKV-Spitzenverband 2018).

\section{Herleitung der Forschungsfragen}

Initial stellt sich die Frage, ob die vereinbarten Notfallstufen mit der Versorgung wesentlicher Notfälle quantitativ und qualitativ korrespondieren. Dies ist jedoch mit dem zumeist auf Abrechnungsdaten basierenden und zur Verfügung stehenden Datenmaterial nicht vollumfänglich zu beantworten, da a) Patienten meist mit (Leit-)Symptomen eine Notaufnahme aufsuchen und die im Nachgang dokumentierten Diagnosen nicht notwendigerweise den initialen Grund des Krankenhausaufenthalts widerspiegeln, b) die Versorgung von einer Vielzahl von Notfällen nicht mit Qualitätsindikatoren zu evaluieren ist, da diese c) entweder (noch) nicht entwickelt oder nicht flächendeckend erhoben werden. Darüber hinaus fehlt weiterhin ein Konzept für die nach diagnostischer Abklärung und ggf. kurzer Intervention ambulant verbleibenden Patienten, die retrospektiv die größte Patientengruppe darstellen, jedoch prospektiv nur schwierig zu identifizieren sind.

Aufgrund dieser Herausforderungen konzentriert sich die vorliegende Auswertung exemplarisch auf die mit breiter Datenbasis hinterlegten Krankheitsbilder Herzinfarkt, Schlaganfall und proximale Femurfraktur mit osteosynthetischer Versorgung und den jeweiligen etablierten Qualitätsindikatoren. Diese werden auf Grundlage der Leistungsdaten der AOK sowie der Angaben aus den strukturierten Qualitätsberichten ausgewertet. Die Qualitätsindikatoren werden in Abhängigkeit der Notfallstufe des behandelnden Krankenhauses betrachtet.

Dadurch sollen insbesondere zwei wichtige Forschungsfragen beantwortet werden:

1. Wie viele Krankenhäuser werden der jeweiligen Notfallstufe zugeordnet und wie viele Patienten mit Schlaganfall, Herzinfarkt oder Femurfraktur werden in den Häusern verschiedener Notfallstufen behandelt?

2. Ist ein Zusammenhang zwischen den Notfallstufen und den Ergebnissen der ausgewählten Qualitätsindikatoren erkennbar?

\subsection{Methodik}

Für die drei Krankheitsbilder Herzinfarkt, Schlaganfall und proximale Femurfraktur werden risikoadjustierte Ergebnis- und Prozessindikatoren auf der 
Krankenhausebene mit der jeweiligen Notfallstufe des Krankenhauses verknüpft. Deskriptive Statistiken wie Häufigkeitsverteilungen und Korrelationen werden verwendet, um die Zuordnung der Fälle zu den Notfallstufen zu untersuchen und den Einfluss struktureller Unterschiede auf die Indikatoren zu analysieren.

\subsubsection{Daten}

Zur Beantwortung der Forschungsfragen werden verschiedene aggregierte Datenquellen herangezogen:

- Angaben zu den Notfallstufen: Die Informationen zu den Notfallstufen aller deutschen Akutkrankenhäuser wurden über das Wissenschaftliche Institut der AOK (WiDO) und den GKV-Spitzenverband bezogen. Die Daten enthalten für das Jahr $2017 \mathrm{zu}$ jedem zugelassenen Krankenhaus Informationen über die vorhandenen Fachabteilungen und die zugewiesene Notfallstufe. Die Zuordnung zu einer Notfallstufe wird auf Basis der Ausstattung des Krankenhauses zwischen dem Krankenhaus und den Krankenkassen individuell verhandelt. Da zum Zeitpunkt der Analyse noch nicht alle Verhandlungen abgeschlossen waren, wird für Krankenhäuser ohne Verhandlungsergebnis die auf Basis der vorhandenen Fachabteilungen im Jahr 2017 prognostizierte Notfallstufe herangezogen.

- Daten aus dem QSR-Verfahren: Informationen aus dem Verfahren „Qualitätssicherung mit Routinedaten (QSR) “basieren auf Abrechnungsdaten stationär behandelter Patienten (vgl. www.qualitaetssicherung-mit-routinedaten.de). Da es sich um Daten der AOK handelt, ist zu berücksichtigen, dass ausschließlich Daten ihrer eigenen Versichertengemeinschaft enthalten sind. Entsprechend bilden diese Daten eine Teilpopulation mit durchschnittlich ca. 1/3 aller Behandlungsfälle ab.

Die Abrechnungsdaten werden gemäß $\$ 301$ SGB V von den Krankenhäusern an die Krankenkassen im Zuge der Abrechnung übermittelt. Sie beinhalten Angaben zu Haupt- und Nebendiagnosen, Operationen und sonstigen Prozeduren, deren Dokumentation durch die Deutschen Kodierrichtlinien geregelt ist. Im QSR-Verfahren werden diese Daten zur Berechnung verschiedener Qualitätsindikatoren herangezogen. Für die ausgewählten Indikatoren wurden für jedes Krankenhaus das Indikatorergebnis sowie die zugrundeliegende Fallzahl (Zähler und Nenner) vom Wissenschaftlichen Institut der AOK bereitgestellt. Die Daten aus dem QSR-Verfahren beziehen sich auf das Jahr 2017.

- Daten der strukturierten Qualitätsberichte der Krankenhäuser: Die Krankenhäuser in Deutschland sind auf der Grundlage des $\mathbb{1} 136 \mathrm{~b}$ Abs. 1 Nr. 3 SGB V verpflichtet, jährlich strukturierte Qualitätsberichte (SQB) zu veröffentlichen. Die zu berichtenden Inhalte legt der G-BA im Auftrag des Gesetz- 
gebers fest. Die Berichte beinhalten Angaben zu Strukturen, Leistungen und Qualität der Krankenhäuser. Enthalten sind beispielsweise Fallzahlen je Diagnose und Prozedur sowie ausgewählte Ergebnisse aus der externen stationären Qualitätssicherung gemäß $\$ 136$ Abs. 1 SGB V. In der vorliegenden Analyse werden die frei zugänglichen Qualitätsberichtsdaten des Jahres 2017 herangezogen.

\subsubsection{Krankheitsbilder und Qualitätsindikatoren}

Für die Analyse wurden die Krankheitsbilder akuter Herzinfarkt, akuter Schlaganfall und proximale Femurfraktur ausgewählt, da diese häufige Notfallbehandlungen in Krankenhäusern darstellen und da für deren adäquate Behandlung spezifische strukturelle Ausstattungen erforderlich sind. Tabelle 1 zeigt eine Übersicht der analysierten Qualitätsindikatoren zu diesen Krankheitsbildern.

\section{Akuter Herzinfarkt}

In Deutschland werden pro Jahr mehr als 200.ooo Personen aufgrund eines akuten Herzinfarktes im Krankenhaus behandelt; im Jahr 2017 verstarben 8, 2\% der stationär aufgenommenen Herzinfarktpatienten während ihres Krankenhausaufenthaltes (Nimptsch u. Mansky 2020).

Entscheidend für das Überleben von Herzinfarktpatienten ist eine zeitnahe und leitliniengerechte Versorgung in einer Einrichtung mit adäquater personeller und technischer Ausstattung. Gerade beim ST-Streckenhebungsinfarkt (STEMI) ist beispielweise eine möglichst frühzeitige Wiedereröffnung der Herzkranzgefäße, bevorzugt mit primärer Linksherzkatheterintervention (perkutane koronare Intervention, PCI), entscheidend für das Behandlungsergebnis (Ibanez et al. 2018). Voraussetzung dafür ist das Vorhandensein eines Linksherzkathetermessplatzes mit 24-Stunden-Verfügbarkeit, welcher eine Anforderung für die Zuweisung der Notfallstufen 2 und 3 ist, jedoch auch in Krankenhäusern ohne Notfallstufe, aber mit einer sog. Chest Pain Unit

\section{Tab. 1 Analysierte Qualitätsindikatoren}

\begin{tabular}{|c|c|c|}
\hline Krankheitsbild & Qualitätsindikator & $\begin{array}{l}\text { Daten- } \\
\text { quelle }\end{array}$ \\
\hline Herzinfarkt & Sterblichkeit innerhalb von 30 Tagen bei Herzinfarkt & QSR \\
\hline Schlaganfall & $\begin{array}{l}\text { Sterblichkeit innerhalb von } 30 \text { Tagen bei Hirninfarkt oder } \\
\text { intrazerebraler Blutung }\end{array}$ & QSR \\
\hline \multirow{2}{*}{$\begin{array}{l}\text { Proximale Femurfraktur } \\
\text { mit osteosynthetischer } \\
\text { Versorgung }\end{array}$} & Präoperative Verweildauer > 24 Stunden & SQB \\
\hline & $\begin{array}{l}\text { Verhältnis der beobachteten zur erwarteten Rate ( } 0 / E) \\
\text { an Todesfällen }\end{array}$ & SQB \\
\hline
\end{tabular}


(spezialisierte Einheit zur Diagnostik und Therapie von Notfällen mit Herzinfarktsymptomen) vorhanden ist.

In der Kardiologie existieren zahlreiche Ergebnis- und Prozessindikatoren, die auf die Struktur des Krankenhauses und die Behandlungsqualität schließen lassen. Zur Bewertung der Versorgung wurde der Ergebnisqualitätsindikator „Sterblichkeit innerhalb von 30 Tagen bei Herzinfarkt“ aus dem QSR-Verfahren ausgewertet. Dieser Indikator beschreibt die risikoadjustierte Sterblichkeit von AOK-versicherten Herzinfarktpatienten (Hauptdiagnose I21 oder I22) innerhalb von 30 Tagen nach der Krankenhausaufnahme und wird als Verhältniszahl von beobachteten $(\mathrm{O})$ zu erwarteten $(\mathrm{E})$ Fällen dargestellt $(\mathrm{O} / \mathrm{E})$.

\section{Modul Durchblutungsstörungen am Herzen}

Als Strukturmerkmal wird das Vorhandensein eines Linksherzkathetermessplatzes mit 24-Stunden-Verfügbarkeit betrachtet. Dieses Merkmal ist in den strukturierten Qualitätsberichten im Datenjahr 2017 für 472 Standorte (430 Häuser) enthalten (Datenfeld AA69). Da die Analysen auf Hausebene durchgeführt wurden, wurde insgesamt etwa bei einem Drittel (410 Häuser) der 1.072 Krankenhäuser mit Qualitätsindikator Sterblichkeit bei Herzinfarkt ein 24/7-verfügbarer Linksherzkathetermessplatz notfallstufenübergreifend zugeordnet.

\section{Schlaganfall}

Die Anzahl der Behandlungen aufgrund eines Schlaganfalls in deutschen Krankenhäusern liegt bei ca. 300.000 pro Jahr. Ca. 85\% der Schlaganfälle sind auf eine akute Durchblutungsstörung des Hirngewebes zurückzuführen (Hirninfarkt), während in ca. 15\% der Behandlungen eine Hirnblutung den Schlaganfall verursacht (Nimptsch u. Mansky 2020). Daher ist in der Notfallbehandlung des Schlaganfalls eine rasche bildgebende Diagnostik erforderlich, um die Ursache zu bestimmen und die erforderliche Therapie einzuleiten. Dies erfordert u.a. eine 24-Stunden-Verfügbarkeit der diagnostischen und therapeutischen Ausstattung. Bestimmte therapeutische Maßnahmen, wie z.B. die mechanische Entfernung eines Blutgerinnsels bei Hirninfarkt oder chirurgische Interventionen bei Hirnblutungen, sind nur in spezialisierten Einrichtungen mit entsprechenden Fachabteilungen (Neuroradiologie, Neurochirurgie) möglich. Daher sind bei diesem Krankheitsbild auch häufig Verlegungen von der erstaufnehmenden Einrichtung in ein spezialisiertes Krankenhaus erforderlich. Eine primäre Diagnostik und Versorgung muss in Krankenhäusern der Notfallstufen 2 und 3 rund um die Uhr verfügbar sein. In vielen Krankenhäusern existieren spezialisierte Einheiten für die Versorgung des akuten Schlaganfalls (sog. Stroke Units).

Zur Ergebnisbewertung wurde der Ergebnisqualitätsindikator „Sterblichkeit innerhalb von 30 Tagen bei Hirninfarkt oder intrazerebraler Blutung " aus dem QSR-Verfahren herangezogen, der die risikoadjustierte Sterblichkeit (O/E) be- 
zogen auf AOK-versicherte Patienten darstellt. Der Indikator umfasst die Einschlussdiagnosen Intrazerebrale Blutung (Hauptdiagnose I61), Hirninfarkt (I63) und nicht als Blutung oder Infarkt definierte Schlaganfälle (I64).

\section{Modul Schlaganfallversorgung}

Entsprechend der Regelungen des G-BA wird ein Krankenhaus auch ungeachtet der Anforderungen an die Notfallstufe 1 dem Modul Schlaganfallversorgung zugeordnet, wenn es über eine Stroke Unit verfügt. Das Vorhandensein einer Stroke Unit ist in den strukturierten Qualitätsberichten im Datenjahr 2017 für 406 Standorte (396 Häuser) enthalten (Datenfeld VN24). Eine weitere Möglichkeit zur Ermittlung des Vorhandenseins einer Stroke Unit ist die Höhe der abgerechneten OPS-Codes. So ist mit großer Wahrscheinlichkeit davon auszugehen, dass ein Krankenhaus über eine Stroke Unit (wenn auch nicht zwingend nach den Stroke Unit-Kriterien der Deutschen Schlaganfall-Gesellschaft zertifiziert) verfügt, wenn die OPS-Codes 8-981 (Komplexbehandlung des akuten Schlaganfalls) und 8-98b (Andere neurologische Komplexbehandlung) ${ }^{3}$ mindestens 10-mal in einem Jahr abgerechnet wurden (Nimptsch u. Mansky 2012). Für die Zuordnung einer Stroke Unit werden Daten beider Definitionen verknüpft, sodass über die Herleitung der oben genannten 10 OPS-Codes 463 Krankenhäuser, ergänzt um 18 Krankenhäuser mit einer Stroke Unit laut Qualitätsbericht notfallstufenübergreifend zugeordnet wurden.

\section{Proximale Femurfraktur mit osteosynthetischer Versorgung}

Jährlich werden in Deutschland circa 150.000 Patienten aufgrund einer proximalen Femurfraktur stationär behandelt (Nimptsch u. Mansky 2020; Statistisches Bundesamt 2017). Die Frakturen treten überwiegend bei älteren Personen auf und müssen meist operativ behandelt werden. Kann das Hüftgelenk erhalten bleiben und muss nicht durch eine Endoprothese ersetzt werden, so spricht man von einer osteosynthetischen Behandlung. Bei dieser wird, sofern es die Lokalisation der Fraktur erlaubt, z.B. ein Marknagel in den betroffenen Knochen eingeführt. Diese Behandlung sollte nach einem Unfall möglichst zeitnah durchgeführt werden, da neben der Komplikationsrate auch die Sterblichkeitsrate erheblich steigt $(13,9 \%$ bei erfolgtem Eingriff innerhalb von 24 Stunden gegenüber 33,3\% bei späterer Versorgung), wenn zu lange mit der Operation gewartet wird (IQTIG 2018).

In der vorliegenden Analyse wurde der Anteil der Patienten mit präoperativer Verweildauer > 24 Stunden als Prozessindikator betrachtet, der im Rahmen der externen stationären Qualitätssicherung in allen Krankenhäusern erhoben und im strukturierten Qualitätsbericht veröffentlicht wird. Weiterhin wurde als Ergebnisqualitätsindikator die risikoadjustierte Sterblichkeit (O/E) betrachtet. Fachabteilungen mit einer Fallzahl oder einem Zähler von unter ein bis drei Patienten veröffentlichen aus Datenschutzgründen kein Indikatorergebnis zu diesen Fällen, weshalb diese nicht ausgewertet wurden. 


\subsubsection{Zuordnung der Notfallstufen}

Im Jahr 2019 begannen die Verhandlungen von Notfallstufen mit den an der Notfallversorgung teilnehmenden Krankenhäusern. Bis zum Zeitpunkt der Finalisierung der vorliegenden Analyse waren jedoch noch nicht alle Verhandlungen abgeschlossen. Daher wurden zwei Datenquellen für die Zuordnung der Notfallstufen verwendet:

- die bereits verhandelten Notfallstufen von 2019 sowie

- eine vom GKV-Spitzenverband initiierte und validierte Notfallstufen-Prognose für das Jahr 2018 basierend auf Daten aus dem Jahr 2017

Sofern vorhanden, wurden für die Auswertung die verhandelten Notfallstufen herangezogen, ansonsten aber die prognostizierten Notfallstufen verwendet. Eine verhandelte Notfallstufe war für ca. die Hälfte der Krankenhäuser bekannt. Krankenhäuser mit vorliegendem Qualitätsindikator ohne Angabe zur Notfallstufe wurden in der Auswertung nicht berücksichtigt (Herzinfarkt 26, Schlaganfall 55, Proximale Femurfraktur 163 Krankenhäuser).

Die Stufenzuordnung erfolgte bei den verhandelten als auch den prognostizierten Notfallstufen auf der Krankenhausebene (über das Institutionskennzeichen). Dafür wurde die jeweils höchste Notfallstufe der Erwachsenenversorgung aller zu einem Institutionskennzeichen zugehörigen Standorte herangezogen. Da die Qualitätsindikatoren teilweise nicht standortbezogen, sondern nur für Institutionskennzeichen der Krankenhäuser vorlagen, wurde der Großteil der Analysen standortunabhängig auf die Institutionskennzeichen bezogen.

Die Krankenhäuser wurden entsprechend einer der drei Notfallstufen $(1,2,3)$ oder der Nichtteilnahme (gekennzeichnet mit der Ziffer „, “) zugeordnet. Krankenhäuser ohne Notfallstufe, die an den Modulen Schlaganfallversorgung und/ oder Durchblutungsstörungen am Herzen teilnehmen, werden unter „o*“ ausgewiesen. In den Datenblättern ist zusätzlich der Anteil von Krankenhäusern mit einem ständig verfügbaren Linksherzkathetermessplatz sowie einer Stroke Unit gekennzeichnet $\left(1^{*}, 2^{*}, 3^{*}\right)$.

\subsection{Ergebnisse}

Folgende Anzahl an Krankenhäusern und Behandlungsfällen wurden ausgewertet:

- Herzinfarkt: 30-Tages-Sterblichkeit: 1.072 Krankenhäuser (mit 64.920 AOK-versicherten Behandlungsfällen)

- Schlaganfall: 30-Tages-Sterblichkeit: 1.124 Krankenhäuser (mit 86.386 AOK-versicherten Behandlungsfällen)

- Proximale Femurfraktur mit osteosynthetischer Versorgung: Prä-operative Verweildauer > 24 h: 759 Krankenhäuser mit 52.656 Behandlungsfällen; Verhältnis (O/E) an Todesfällen: 449 Krankenhäuser mit Fallzahlangabe mit ins- 
gesamt 30.053 Behandlungsfällen und 376 Krankenhäuser ohne Fallzahlangabe.

\subsubsection{Herzinfarkt}

\section{Qualitätsindikator Sterblichkeit innerhalb von 30 Tagen}

Die Hälfte der Krankenhäuser, für die die Sterblichkeit bei Herzinfarkt ausgewertet wurde, ist der Notfallstufe 1 zuzuordnen. Der Großteil der Behandlungsfälle verteilt sich relativ gleichmäßig auf die Notfallstufen 1, 2 und 3 (s. Abb. 1). Mehr als die Hälfte der Patienten wird in Häusern der höheren Notfallstufen 2 und 3 behandelt.

In Krankenhäusern der Notfallstufe 1 (ohne Linksherzkathetermessplatz) wurden im Jahr 2017 durchschnittlich 19 Fälle behandelt und somit deutlich weniger als in allen Krankenhäusern, die über die zusätzliche apparative Ausstattung verfügen. Ungefähr jedes siebte Krankenhaus (14\%) nimmt nicht an der Notfallversorgung teil, hier wurden mit durchschnittlich 4 Behandlungsfällen die wenigsten Fälle pro Jahr versorgt. Je höher die Notfallstufe, umso höher war auch der Anteil der Krankenhäuser mit einem 24/7-Linksherzkathetermessplatz (s. Tab. 2). Die Streudiagramm-Darstellung des Indikatorergebnisses zeigt eine hohe Variation des Outcomes, die in den unteren Notfallstufen stärker ausgeprägt ist und mit zunehmender Fallzahl abnimmt (s. Abb. 2).

Auch in der Box-Plot-Darstellung (s. Abb. 3) wird eine hohe Variation, insbesondere bei den Krankenhäusern der Notfallstufe 1, sichtbar.

Wenn das Indikatorergebnis aggregiert auf Notfallstufenebene betrachtet wird, liegt die Sterblichkeit in spezialisierten Häusern der Notfallstufe o* deutlich unter dem Bundesdurchschnitt. In den Häusern mit Notfallstufe 1, 2 und 3 bewegt sich die Sterblichkeit um den Erwartungswert von 1 (s. Tab. 2).

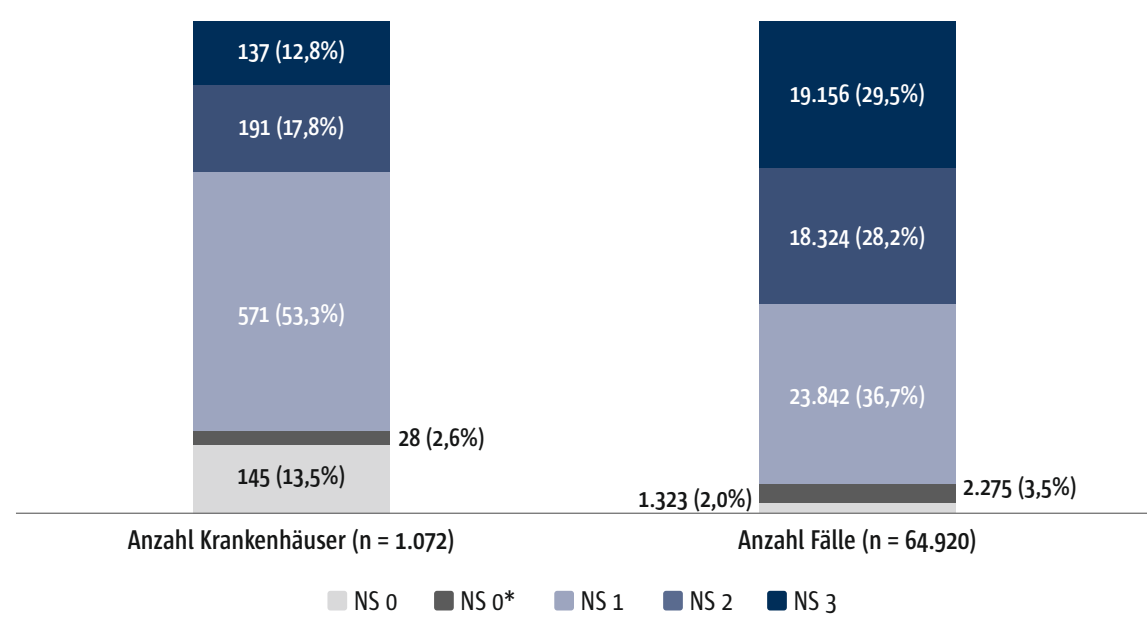

Abb. 1 Anzahl (Anteil) der Krankenhäuser und Fälle nach Notfallstufen 


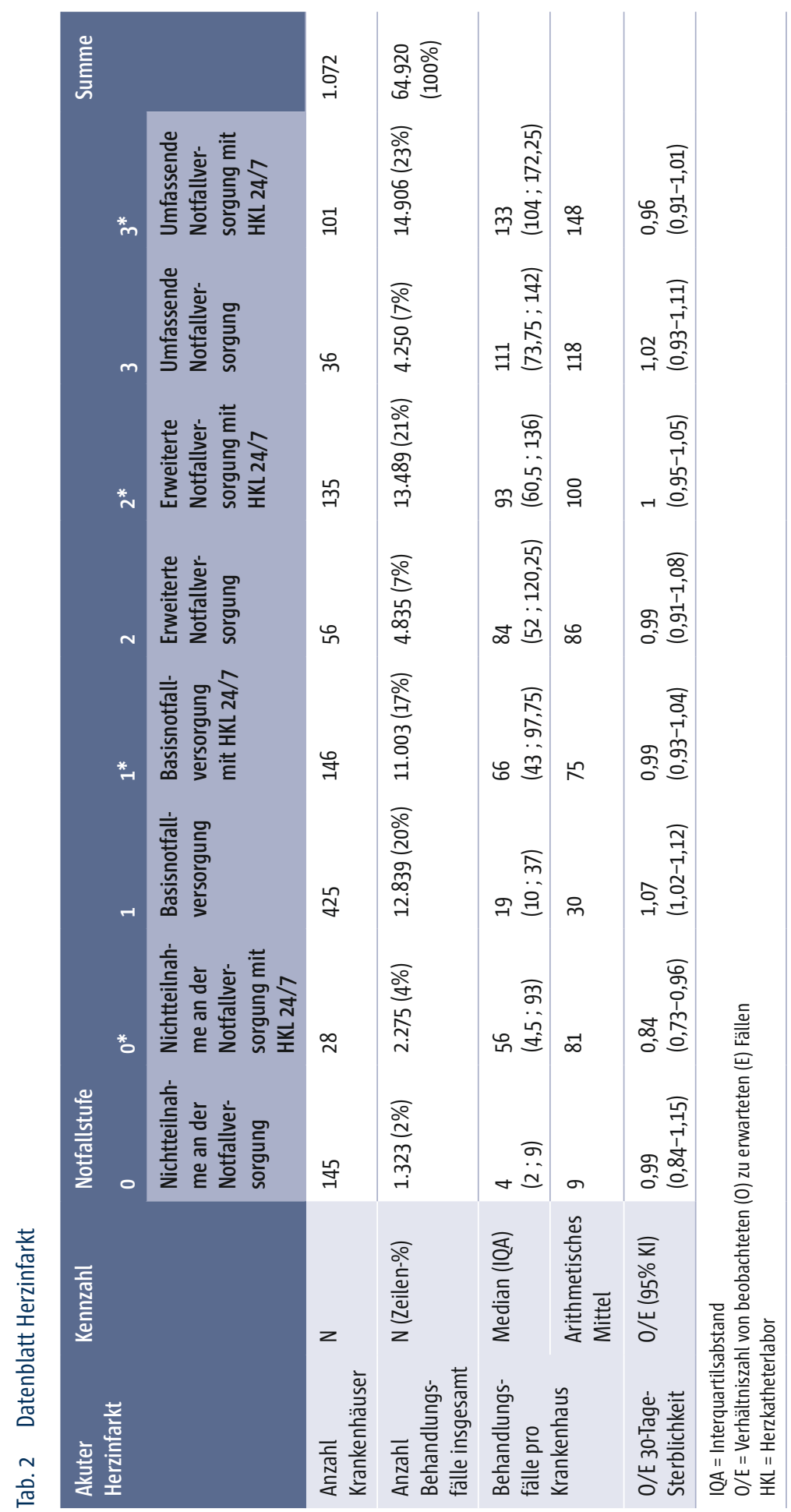


3 Notfallstufen: qualitative Unterschiede in der Notfallversorgung

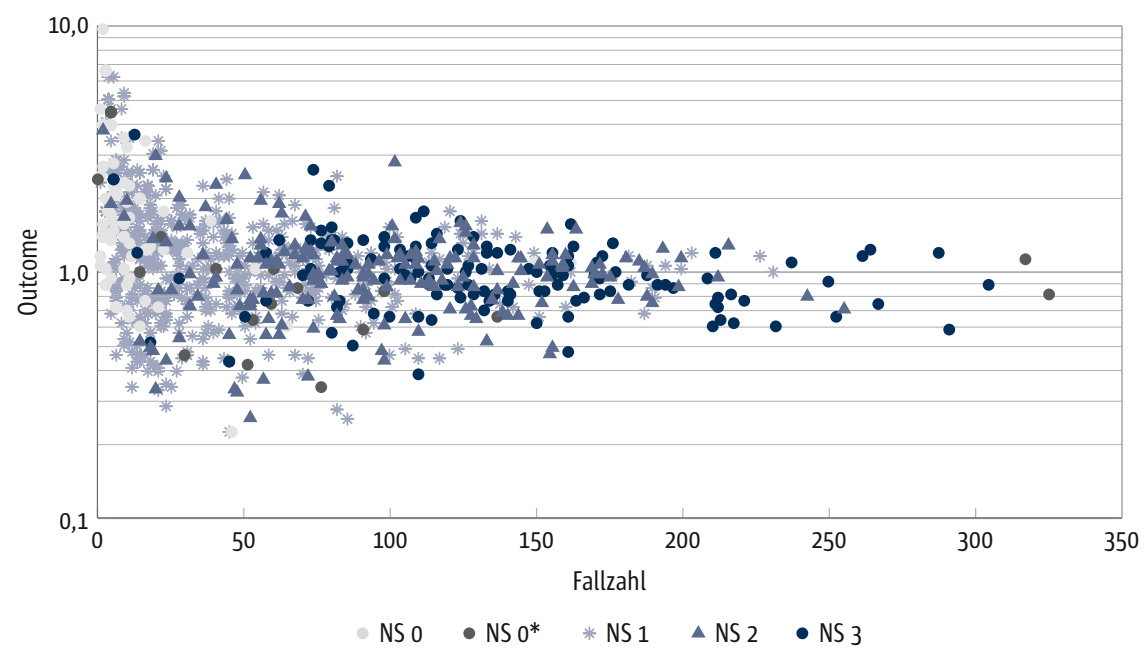

Abb. 2 30-Tage-Sterblichkeit bei Herzinfarkt: Verhältnis der beobachteten (0) zu erwarteten (E) Todesfällen (Streudiagramm) (Krankenhäuser mit > 350 Fällen: N = 3, 1 x NS 3, 1 x NS 0*, $1 \times$ NS 1)

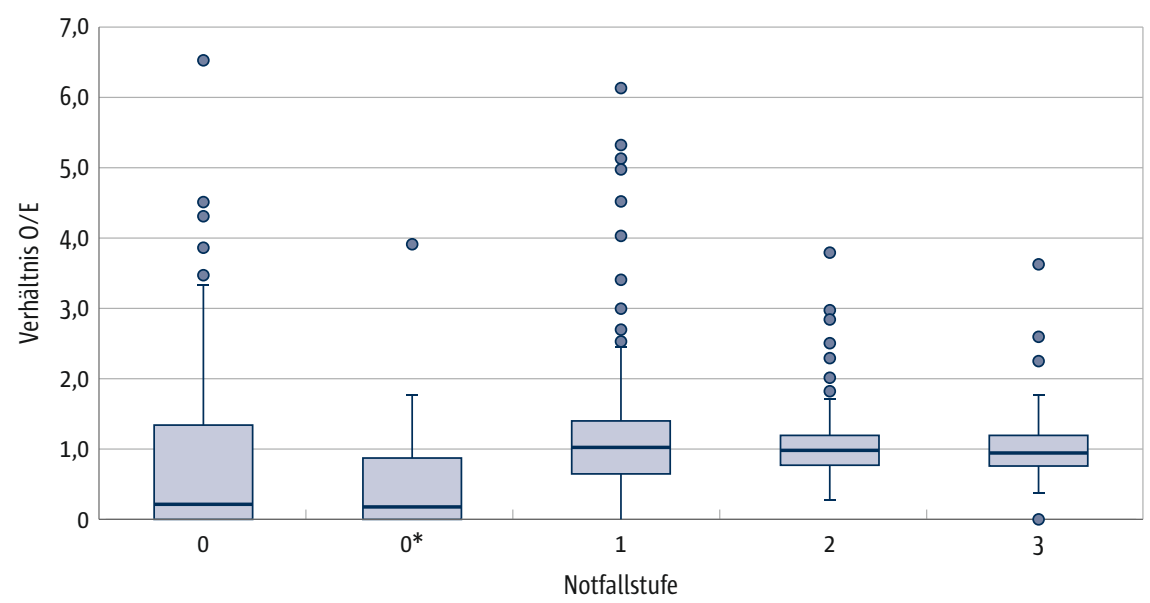

Abb. 3 30-Tage-Sterblichkeit bei Herzinfarkt: Verhältnis der beobachteten (0) zu erwarteten (E) Todesfällen (Box-Plot)

\subsubsection{Schlaganfall}

\section{Qualitätsindikator Sterblichkeit innerhalb von 30 Tagen}

Für die Sterblichkeit bei Schlaganfall wurden 1.124 Krankenhäuser mit ca. 86.0oo AOK-versicherten Behandlungsfällen ausgewertet. Die Hälfte der Krankenhäuser, die Schlaganfallpatienten versorgen, sind der Notfallstufe 1 


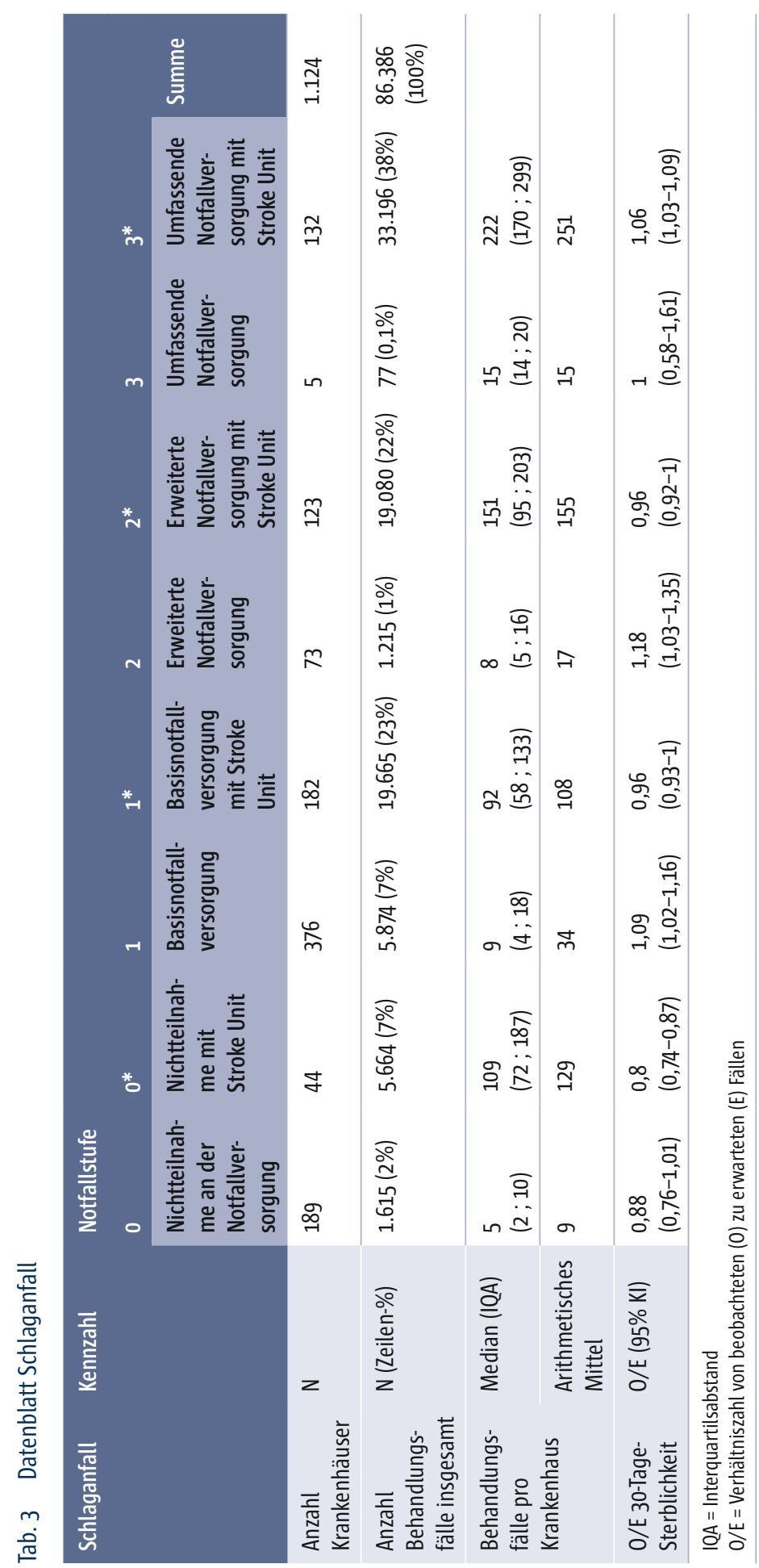


zuzuordnen. Ein Großteil der Patienten wird jedoch in der höchsten Notfallstufe 3 behandelt, wo beinahe alle Krankenhäuser über eine Stroke Unit verfügen.

Knapp 7\% der Behandlungsfälle werden in den spezialisierten Krankenhäusern mit Modul Schlaganfallversorgung versorgt (s. Tab. 3). Dort werden im Durchschnitt deutlich mehr Fälle pro Jahr (109) als in Krankenhäusern der Notfallstufe 2 (8) und 3 (15) behandelt, die nicht über eine Stroke Unit verfügen.

Die Darstellung im Streudiagramm (s. Abb. 5) zeigt, dass die Krankenhäuser innerhalb der Notfallstufen in ihrer Fallzahl stark variieren. Während sich

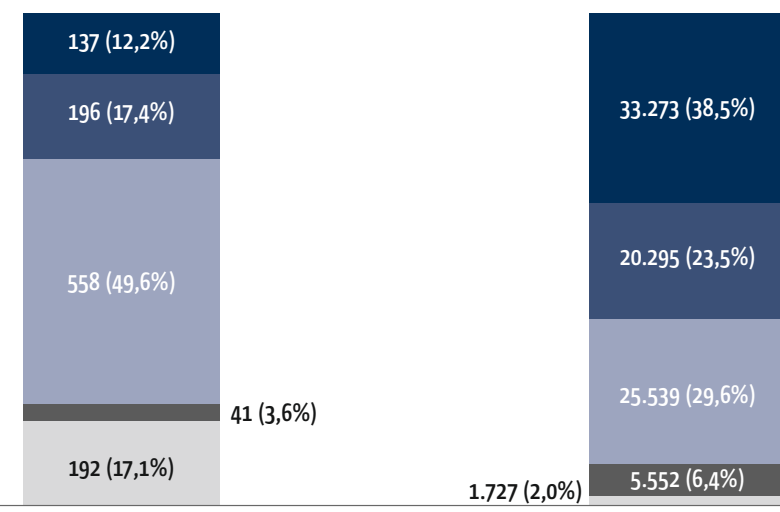

Anzahl Krankenhäuser $(\mathrm{n}=1.124)$

Anzahl Fälle $(n=86.386)$

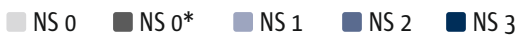

Abb. 4 Anzahl (Anteil) der Krankenhäuser und Fallzahlen nach Notfallstufen

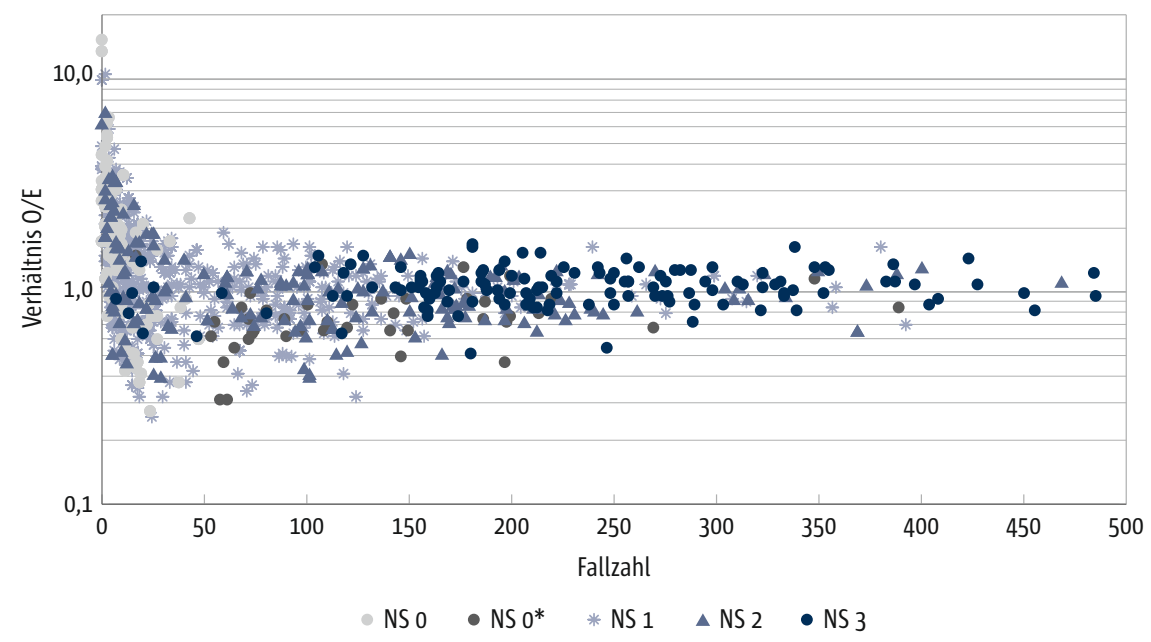

Abb. 5 30-Tage-Sterblichkeit bei Schlaganfall: Verhältnis der beobachteten (0) zu erwarteten (E) Todesfällen (Streudiagramm) 


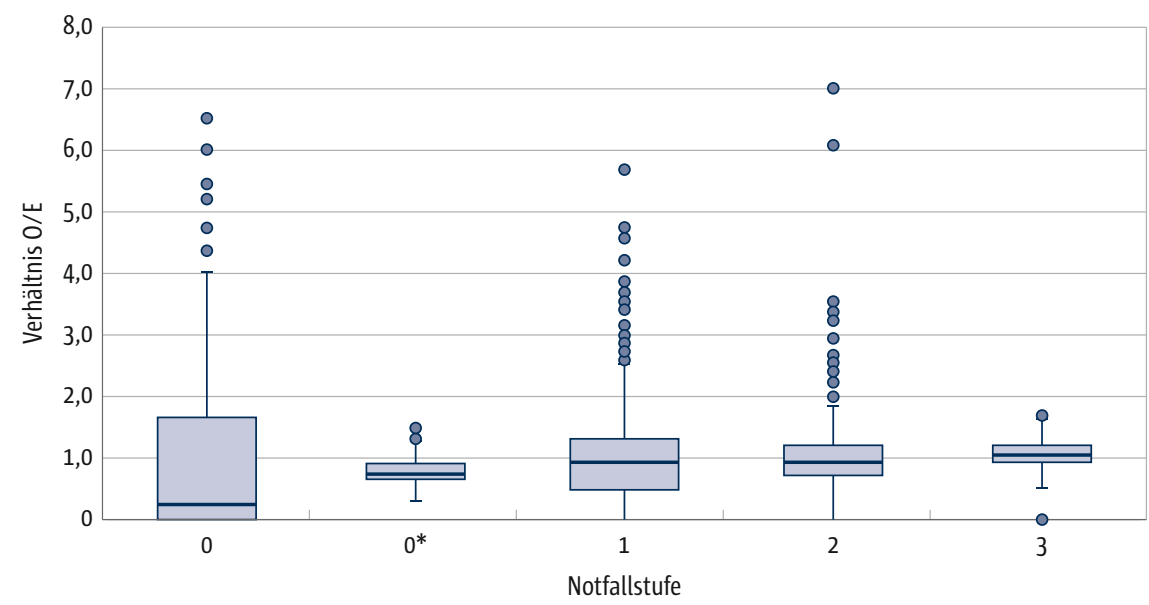

Abb. 6 30-Tage-Sterblichkeit bei Schlaganfall: Verhältnis der beobachteten (0) zu erwarteten (E) Todesfällen (Box-Plot)

beim Herzinfarkt die Häuser der Notfallstufe 1 deutlicher zwischen Fallzahlen von 1 bis 50 bewegen, so ist die Streuung beim Schlaganfall breiter und es gibt mehr Häuser der Notfallstufe 1 und 2, die insgesamt höhere Fallzahlen haben. Es lässt sich eine breite Streuung des Ergebnisses bei nicht an der Notfallversorgung teilnehmenden Krankenhäusern (s. Abb. 6) erkennen. Wie schon beim Herzinfarkt wird ebenfalls eine vergleichsweise hohe Qualitätsvariation der Krankenhäuser in Notfallstufe 1 deutlich.

Bei Betrachtung des Sterblichkeitsverhältnisses über die Gruppen der Krankenhäuser zeigt sich vor allem in Krankenhäusern der Notfallstufe 1 (ohne zugeordnete Stroke Unit) und Notfallstufe $3^{*}$ (mit zugeordneter Stroke Unit) eine höhere Sterblichkeit, während diese bei Krankenhäusern ohne Teilnahme (o) sowie mit Stroke Unit (o*) deutlich geringer ist (s. Tab. 3).

\subsubsection{Proximale Femurfraktur mit osteosynthetischer Versorgung}

\section{Qualitätsindikator prä-operative Verweildauer $>24 \mathrm{~h}$}

Der Qualitätsindikator wurde für 759 Krankenhäuser mit insgesamt ca. 52.0oo Behandlungsfällen ausgewertet. Wie bei Herzinfarkt und Schlaganfall ist ein Großteil der Häuser der Notfallstufe 1 zugeordnet (55\%). Die Behandlungsfälle werden ebenfalls meist in der Stufe 1 versorgt, gefolgt von Stufe 3 und 2 (s. Abb. 7).

Die Anzahl der Behandlungsfälle je Haus steigt kontinuierlich mit zunehmender Notfallstufe - von circa 37 Fällen in Häusern, die nicht an der Notfallversorgung teilnehmen, auf 104 Fälle in Häusern mit der Notfallstufe 3.

Der Prozessindikator der präoperativen Verweildauer hat als Qualitätsziel vorgesehen, dass $85 \%$ der betroffenen Patienten innerhalb von 24 Stunden operiert 


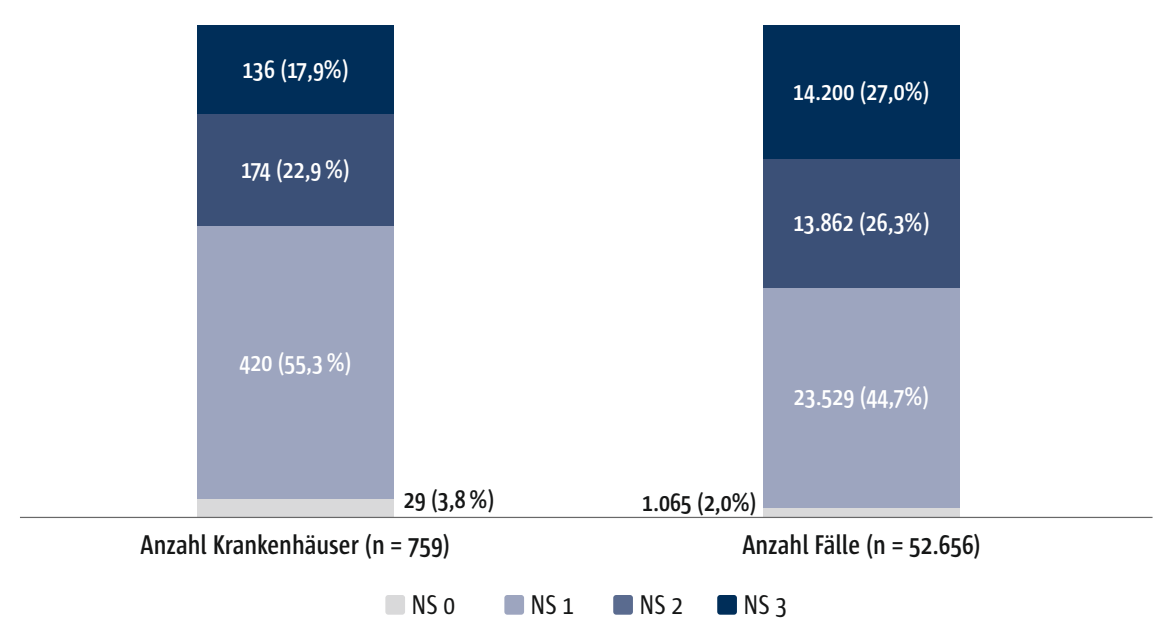

Abb. 7 Proximale Femurfraktur mit osteosynthetischer Versorgung, präoperative Verweildauer $>24$ h: Anzahl der Krankenhäuser und Fallzahlen nach Notfallstufen

werden sollen. Der Referenzbereich (15\%) wird in allen drei Notfallstufen überschritten. Vor allem Häuser, die nicht an der Notfallversorgung teilnehmen, schneiden hier auffällig schlecht ab (knapp 22\%), gefolgt von Häusern der Notfallstufe 3 (knapp 19\%; s. Tab. 4).

\section{Qualitätsindikator Verhältnis (0/E) an Todesfällen}

Für die Ermittlung des Verhältnisses an Todesfällen lagen Daten von Krankenhäusern $(\mathrm{N}=449)$ und Standorten ( $\mathrm{N}=416$, entsprechend 376 Häusern) vor, welche sich anteilig ähnlich über die verschiedenen Stufen verteilen. Wie im Methodik-Teil beschrieben, ist der Grund für diese separierte Betrachtung der, dass für einen Großteil der Standorte lediglich ein Outcome (Verhältnis O/E), jedoch keine Fallzahlen vorliegen.

Ausgewertet wurden ca. 30.000 berichtete Behandlungsfälle in 449 Krankenhäusern sowie die Outcomes der 376 Häuser ohne Fallzahlübermittlung (s. Abb. 8).

Über die Hälfte der Krankenhäuser sind der Notfallstufe 1 zugeordnet. Berücksichtigt man in der Auswertung nur die Angaben der Häuser mit veröffentlichter Fallzahl, so wird erkennbar, dass die durchschnittliche Anzahl der Behandlungsfälle mit steigender Notfallstufe ansteigt (von 21 Fällen bei Notfallstufe o bis zu 103 Fällen bei Notfallstufe 3, s. Tab. 4). Beinahe ein Drittel der Krankenhäuser weist ein Indikatorergebnis (O/E) von o auf - insgesamt sind es 201 Standorte. Das bedeutet, dass dort kein Patient bei einem osteosynthetischen Eingriff nach Femurfraktur innerhalb von 30 Tagen verstorben ist.

Der Referenzbereich des Indikators in der Bundesauswertung liegt bei $\leq 2,02$ (90. Perzentil) (IQTIG 2018). Dieser wurde im Mittel bei keiner der Notfallstufen überschritten (s. Abb. 9). 


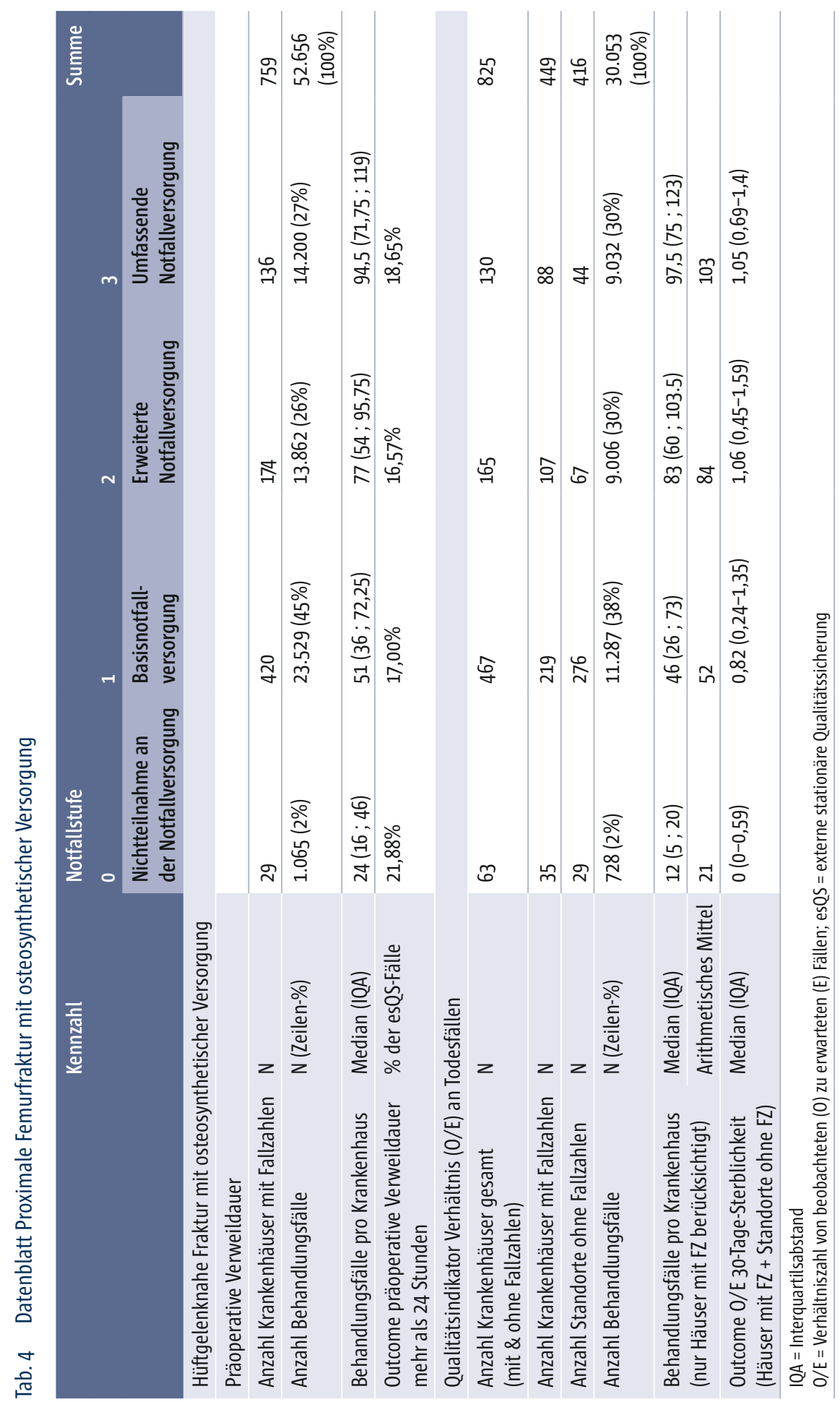




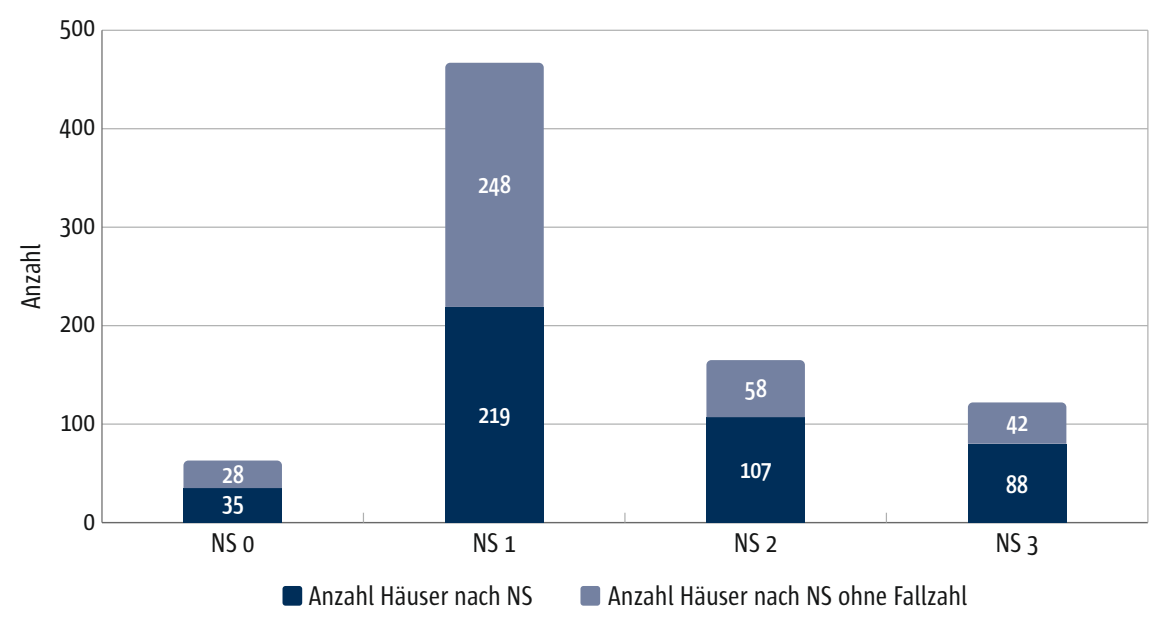

Abb. 8 Proximale Femurfraktur mit osteosynthetischer Versorgung, Verhältnis (0/E) an Todesfällen: Anzahl der Krankenhäuser mit (449) und ohne (376) Fallzahlübermittlung nach Notfallstufen

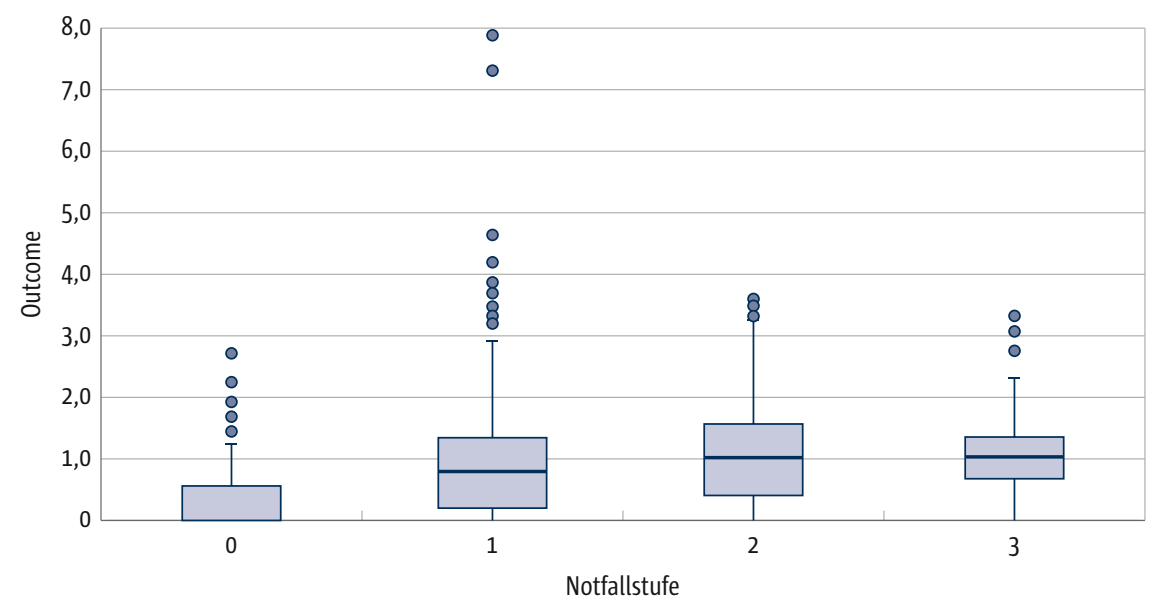

Abb. 9 Proximale Femurfraktur mit osteosynthetischer Versorgung, Verhältnis (0/E) an Todesfällen: Verhältnis der beobachteten (0) zu erwarteten (E) Fällen (Box-Plot)

\subsection{Diskussion}

Die vorliegende Arbeit analysiert Versorgungsdaten für drei typische, i.d.R. stationär versorgte Notfallbehandlungen (Herzinfarkt, Schlaganfall, proximale Femurfraktur). Für alle drei Krankheitsbilder sind Qualitätsindikatoren verfügbar, die eine Bewertung der Versorgung ermöglichen.

Dabei stellen diese Fälle quantitativ nur einen Ausschnitt des Notfallaufkommens in den Notaufnahmen dar. 
Die Therapie dieser Krankheitsbilder findet regelhaft außerhalb der Notaufnahme in den entsprechenden Fachabteilungen statt (Ausnahme Lysetherapie bei Schlaganfall, abhängig von der lokalen Organisation). Insofern dienen die drei Krankheitsbilder mehr der Charakterisierung der Leistungsfähigkeit einer entsprechenden Fachabteilung im Zusammenspiel mit der Notaufnahme, die maßgeblich für die Ersteinschätzung, Primärdiagnostik und das Anstoßen interner Alarmpfade zuständig ist.

\section{Herzinfarkt}

Die Auswertungen zum Herzinfarkt zeigen, dass Krankenhäuser der Notfallstufen 0,1 und 3 mit einem 24/7-verfügbaren Linksherzkathetermessplatz bessere Qualitätsergebnisse, d.h. eine deutlich geringere Sterblichkeit, aufweisen. Bei Krankenhäusern der Notfallstufe 2 ist der Unterschied zwischen Krankenhaus mit apparativer Ausstattung $\left(2^{*}\right)$ und ohne geringfügig. Die spezialisierten Krankenhäuser ohne Notfallstufe $\left(\mathrm{o}^{*}\right)$ versorgen nur einen kleinen Anteil der betroffenen Patienten. Über ein Drittel aller Fälle werden in Häusern mit der Notfallstufe 1 behandelt, von denen nur wenige über einen ständig verfügbaren Linksherzkathetermessplatz verfügen. Sichtbar ist außerdem eine Zunahme der Streuung der Ergebnisse mit abnehmender Versorgungsstufe. Das gute Ergebnis der spezialisierten „Modulkliniken“ (Notfallstufe o*) könnte zum einen darin begründet sein, dass hierzu mehrheitlich Herzzentren zählen, die überwiegend Patienten mit eindeutiger Symptomatik zugewiesen bekommen. Zum anderen verfügen diese Kliniken nachweislich über einen Linksherzkathetermessplatz und somit über die notwendige Infrastruktur für eine adäquate Behandlung. Für letztere Interpretation spricht auch das gute Ergebnis der Krankenhäuser mit Notfallstufe 2 und 3, von denen zwei Drittel aller Kliniken mit einem Linksherzkathetermessplatz ausgestattet sind. Dass dort die Streuung der Ergebnisse geringer ist, entspricht den Beobachtungen vorangegangener Studien von Mengen-Ergebnis-Zusammenhängen in der Herzinfarktversorgung (Bolczek et al. 2019; Nimptsch u. Mansky 2017).

Deutlich ist insbesondere die Qualitätsvariation bei Krankenhäusern der Notfallstufe 1, von denen wie erwähnt nur ein vergleichsweise geringer Anteil über einen ständig verfügbaren Linksherzkathetermessplatz verfügt, die jedoch mehr als ein Drittel aller Herzinfarktpatienten behandeln.

Bei der Interpretation der Ergebnisse ist zu bedenken, dass bei der Zuweisung der Patienten in die Krankenhäuser auch Selektionseffekte (z.B. Selbsteinweisungen, Transportentscheidungen des Rettungsdienstes) vorliegen könnten, die jedoch in der vorliegenden Analyse nicht untersucht werden konnten.

\section{Welche Daten werden benötigt, um die Qualität der Notfallversorgung beim Herzinfarkt zu bewerten?}

Grundsätzlich scheint es schwierig, die Qualität der Notfallversorgung allein auf der Ebene der Diagnose zu ermitteln. Tatsächlich müssen patientenbezo- 
gene Variablen und hier insbesondere die primäre Symptomatik, Begleiterkrankungen, Vormedikation und Vitalparameter einbezogen werden, um homogene Patientengruppen zu definieren, deren Versorgung in einem Benchmark bewertet werden kann. Patienten werden selten mit einer verlässlichen Diagnose und regelhaft mit einem oftmals schwer zu fassenden Symptomkomplex eingeliefert. So zeigt sich, dass der Infarkt zwar oft zu Brustschmerzen führt, die das häufigste einzelne Leitsymptom in der Notaufnahme sind; von allen Vorstellungen aufgrund von Brustschmerzen haben dann jedoch nur 10\% einen bestätigten Herzinfarkt (Möckel et al. 2013).

Primäre Daten der Notfallversorgung stehen bisher in Routinedaten nur sehr eingeschränkt zur Verfügung, zumal allein die Lokalisation der Versorgung in einer Zentralen Notaufnahme nur indirekt erschlossen werden kann. Projekte wie das Notaufnahmeregister AKTIN, das aus der Routinedokumentation des DIVI-Notaufnahmedatensatzes Daten automatisiert extrahiert, bilden einen Ansatz, wie zukünftig Daten generiert werden können (Kulla et al. 2016). Allerdings nehmen bisher nur sehr wenige Kliniken (niedriger zweistelliger Bereich) an diesem Register teil, dessen IT-Integration kostenintensiv ist. Hier sind allerdings im Rahmen des Nationalen Forschungsnetzes der Universitätsmedizin zu COVID-19 deutliche Zuwächse 2020/21 absehbar.

Darüber hinaus gibt es bisher kein strukturiertes Qualitätsmanagement im Hinblick auf die Qualität der Routinedatenerhebung in der (möglicherweise überlasteten) Notaufnahme.

\section{Schlaganfall}

Für die Auswertungen zum Schlaganfall gelten ähnliche Überlegungen wie für den Herzinfarkt. 30\% aller Behandlungsfälle werden in Krankenhäusern mit Notfallstufe 1 versorgt, in denen mehrheitlich keine Stroke Unit vorhanden ist.

Auf die Schlaganfallbehandlung spezialisierte Kliniken erzielen das beste Qualitätsergebnis, während überraschenderweise die Krankenhäuser, die nicht an der Notfallversorgung teilnehmen, ebenfalls überdurchschnittlich gut abschneiden. Dem gegenüber stehen die Krankenhäuser der Notfallstufe 1 ohne Stroke Unit, in denen die Sterblichkeit von allen Notfallstufen am höchsten ist. Auch die Häuser der höchsten Notfallstufe mit zugeordneter Stroke Unit weisen ein schlechtes Qualitätsergebnis auf.

Das gute Ergebnis der Krankenhäuser, die nicht an der Notfallversorgung teilnehmen, jedoch über eine Stroke Unit verfügen, ist vermutlich auf die Spezialisierung und die technische bzw. personelle Ausstattung zurückzuführen. Diese Krankenhäuser sind in vielen Fällen neurologische Fachkliniken mit einem spezialisierten neurologischen Versorgungsangebot und stetiger Verfügbarkeit von qualifiziertem Personal und der relevanten Infrastruktur. Fragen wirft das Ergebnis der Krankenhäuser mit höchster Notfallstufe und zugeordneter Stroke Unit auf, hier liegt die Sterblichkeit deutlich über dem 
Bundesdurchschnitt. Eine naheliegende Erklärung durch Weiterverlegungen komplexer Fälle in Krankenhäuser mit höherer Notfallstufe erklärt das vorliegende Ergebnis nicht, da nach QSR-Logik (auf welcher die Daten basieren) der sog. Startfall betrachtet wird. Dieser umfasst alle (akut-)stationären Fälle im Zeitraum von der Aufnahme bis zur ersten Entlassung, inklusive etwaiger Verlegungen. Somit würde ein Todesfall nach „Aufwärtsverlegung“ dem erstaufnehmenden Krankenhaus mit niedrigerer Notfallstufe zugeordnet. Daher lassen die Ergebnisse eher Selektionseffekte vermuten, da komplexere, atypische und multimorbide Fälle bevorzugt in größere Kliniken transportiert werden. Vor allem Patienten mit Vor- und Nebenerkrankungen können oft wegen Kontraindikationen nicht so effektiv behandelt werden, was möglicherweise das schlechtere Ergebnis der großen Versorger erklären könnte. Zusätzlich kann die in den Limitationen geschilderte Zuweisung der Stroke Units zu den im Beitrag untersuchten Krankenhäusern mit Qualitätsindikator eine Begründung des Ergebnisses sein.

\section{Welche Daten werden benötigt, um die Qualität der Notfallversorgung von Schlaganfällen zu bewerten?}

Auch beim Schlaganfall liegt die Versorgungshoheit regelhaft bei der Fachabteilung. Allerdings ist die Grundgesamtheit kleiner, da Patienten mit akuter Lähmung, Sprachstörung etc. deutlich spezifischer einen Schlaganfall nahelegen, als z.B. der Brustschmerz (Möckel et al. 2013). Im Hinblick auf den Schlaganfall sind Qualitätsindikatoren der Notaufnahme im Bereich der frühen Diagnostik zu suchen, die bereits regelhaft bei allen Schlaganfallpatienten erhoben werden. Insbesondere die Zeit bis zur Computertomografie ist hier eine einfache und entscheidende Größe, die allerdings in Routinedaten insofern schwer zu erheben ist, als dass der entsprechende OPS-Code zwar theoretisch zeitgenau dokumentiert wird, aber die Eintreffzeit in einer Zentralen Notaufnahme aktuell noch nicht regelhaft in Routinedaten erkennbar ist. Hier wird plakativ deutlich, dass es einen verpflichtenden Code geben müsste, der einerseits die Behandlung in einer Zentralen Notaufnahme und andereseits die Eintreffzeit abbildet.

\section{Proximale Femurfraktur}

Die Sterblichkeit bei einer osteosynthetisch versorgten proximalen Femurfraktur ist entgegen der Annahme, dass Krankenhäuser mit höherer Notfallstufe bessere Ergebnisse vorweisen, in Häusern der Stufe 1 oder ohne Teilnahme an der Notfallversorgung geringer.

Hingegen ist der Anteil der Patienten mit einer präoperativen Verweildauer jenseits von 24 Stunden in Krankenhäusern der höheren Notfallstufen 1 bis 3 niedriger. 


\section{Welche Daten werden benötigt, um die Qualität der Notfallversorgung von Femurfrakturen zu bewerten?}

Die Behandlung der proximalen Femurfraktur ist ebenfalls sehr stark durch ein fachgesellschaftlich definiertes Qualitätsmanagement gekennzeichent. Dennoch gibt es zusätzliche Aspekte, die auch zukünftig für die Qualitätsbewertung in der Notaufnahme, aber auch der ambulanten Versorgung herangezogen werden können.

Patienten mit proximaler Femurfraktur sind häufig pflegebedürftig, multimorbid und älter. Dies bedeutet, dass die Erfassung der Komorbidität und von operativen Risikofaktoren wichtig ist, um Kliniken überhaupt vergleichen zu können. Zum anderen haben die älteren Menschen mit proximaler Femurfraktur beispielsweise ein sehr hohes Risiko eines akuten Delirs mit verheerenden prognostischen Auswirkungen. Erste Zeichen des Delirs entwickeln sich mitunter bereits auf dem Transportweg (Rosenthal et al. 2020 sowie Knaak et al. 2020).

Die bisherigen Strukturen der Notaufnahme lassen eine adäquate Prophylaxe und frühzeitige Therapie vielfach nicht zu. Bei der Bewertung der Ergebnisse, insbesondere von Häusern ohne perioperative Mortalität ist davon auszugehen, dass hier eine sehr starke Selektion stattfindet. Möglicherweise weiß der Rettungsdienst aus Erfahrung, dass bestimmte Risikopatienten von kleinen Häusern abgewiesen werden. Dies würde zu einer im Detail nicht nachvollziehbaren „Diversion“ führen.

Ansätze, diese Vorgänge transparenter zu machen, sind zum Beispiel die interdisziplinäre digitale Patientenzuweisung (,IVENA eHealth“), deren Einsatz aber bisher nur sehr begrenzt ist. Darüber hinaus gibt es bisher kaum Daten, die die Qualität und Regeltreue der Zuweisung abbilden und mit dem Schweregrad und den Krankenhausdiagnosen der Patienten in Beziehung setzen. Hier sind zukünftig auch mit zunehmender Digitalisierung des Rettungsdienstes weitere Daten zu erschließen.

\section{Limitationen}

Auf einige Limitationen ist zur Einordnung der Ergebnisse gesondert hinzuweisen:

Da zum Analysezeitpunkt die Verhandlungen über die Notfallstufen nicht abgeschlossen waren, wurde für Krankenhäuser ohne Verhandlungsergebnis die auf Basis der vorhandenen Fachabteilungen im Jahr 2017 prognostizierte Notfallstufe herangezogen.

Bei der Auswertung der Herzinfarktdaten ist außerdem zu beachten, dass als Proxy für Krankenhäuser mit dem Modul Durchblutungsstörungen am Herzen (Notfallstufe $o^{*}$ ) lediglich das Vorhandensein eines Linksherzkathetermessplatzes mit 24-Stunden-Verfügbarkeit betrachtet wurde. Für die Vereinbarung des Moduls gibt es jedoch weitere Voraussetzungen, die erfüllt werden müssen. 
Die zuvor beschriebene Limitation ist auch beim Schlaganfall zu beachten. Obgleich das Vorhandensein einer Stroke Unit die Voraussetzung zur Vereinbarung des Moduls Schlaganfallversorgung ist, konnte diese für deutlich mehr Krankenhäuser als lediglich durch die verpflichtende Selbstangabe der Strukturierten Qualitätsberichte durch die Abrechnung der entsprechenden OPSCodes hergeleitet werden. Die Anzahl der Stroke Units könnte so durch die Definition überschätzt sein. Es ist denkbar, dass eine erneute Auswertung mit den dann verhandelten Notfallstufen leicht andere Ergebnisse zeigen würde. Von den 6o.223 Behandlungsfällen mit proximaler Femurfraktur wurden für den Indikator der präoperativen Verweildauer knapp go Prozent (52.656 Fälle) ausgewertet, allerdings liegt das Ergebnis bei allen Notfallstufen über dem Bundeswert von 16,23\%. Begründet könnte dies darin sein, dass es zu einer Verzerrung durch die Datenschutzregelung (laut der ab nur mindestens vier Fällen ein Ergebnis veröffentlicht werden darf) kommt. Krankenhäuser mit wenigen Patienten fallen so nicht unter die Auswertung, was zu einer Verzerrung der Ergebnisse geführt haben mag.

Beim Indikator des Verhältnisses der beobachteten zur erwarteten Rate (O/E) an Todesfällen können aus demselben Grund nur etwa die Hälfte der Krankenhäuser berücksichtigt werden. Das vorliegende Ergebnis könnte darin begründet sein, dass große Kliniken mit wenigen Todesfällen nicht unter die Auswertung fallen. Als zusätzliche Hürde kommt hinzu, dass für Standorte ohne Fallzahlen zwar ein berichteter Outcome (total measured cases) vorliegt, allerdings keine Werte für beobachtete $(\mathrm{O})$ und erwartete $(\mathrm{E})$ Fälle veröffentlicht werden. Eine Angabe des arithmetischen Mittels war so ebenfalls nicht möglich.

\section{Implikationen}

Zusammenfassend lässt sich insbesondere für die in diesem Beitrag untersuchte Herzinfarkt- und Schlaganfallversorgung festhalten, dass im gestuften System von Notfallstrukturen gute Ergebnisse vor allem von spezialisierten Versorgern erzielt werden. Bei allen betrachteten Qualitätsindikatoren werden außerdem bei Häusern derselben Notfallstufe heterogene Ergebnisse (breite Streuung sowohl in Hinblick auf die Anzahl der Fälle als auch der Outcomes) gefunden.

Dies deutet darauf hin, dass die Einteilung der Krankenhäuser in Notfallstufen das Problem der strukturellen Fragmentierung, der kaum vorhandenen Vernetzung und der Intransparenz von Informationen in der Notfallversorgung nur bedingt lösen kann. Dabei haben diese Probleme mehrere Auswirkungen auf die Versorgungsabläufe; Patienten, einweisende Ärzte und der Rettungsdienst verfügen möglicherweise nicht immer über ausreichende Informationen, welches Krankenhaus entsprechend der Symptomatik für eine adäquate Versorgung in Hinblick auf die personelle und infrastrukturelle Ausstattung am besten geeignet ist. Zudem ist den Daten nicht zu entnehmen, auf welchem Weg, d.h. per Selbsteinweisung, mit dem Rettungsdienst oder durch 
Einweisung eines niedergelassenen Arztes, der Patient in die Notfallaufnahme kommt. Dabei wäre es wünschenswert, dass die Patientensteuerung im Notfall möglichst zielsicher erfolgen kann und dass transparente Informationen über die aktuelle Ausstattung der Krankenhäuser, sowohl für selbst einweisende Patienten als auch für einweisende Ärzte sowie den Notarzt- und Rettungsdienst, zur Verfügung stehen. Eine optimierte Rettungskette, die insbesondere auf diesen transparenten Informationen beruht, würde vermutlich zu weniger Verlegungen und einer geringeren Sterberate führen.

Dass die spezialisierten Kliniken in der Untersuchung vergleichsweise sehr gute Ergebnisse erzielen, deutet darauf hin, dass auch andere Merkmale als die reine Größe eines Krankenhauses entscheidend für die Ergebnisqualität sind. Allerdings ist hinzuzufügen, dass eine Patientenselektion bei der primären Präsentation (typisch versus atypisch) nicht auszuschließen ist und komplexere Fälle vermutlich häufiger bei den größeren Versorgern behandelt werden. Unterschiede in der Patientenzusammensetzung werden zwar bei der Berechnung der O/E-Indikatoren für die Sterblichkeit berücksichtigt, allerdings hat die Risikoadjustierung allein auf Basis der Abrechnungsdaten Schwächen und es können naturgemäß nicht alle Patientencharakteristika berücksichtigt werden. Das Outcome könnte daher maßgeblich durch die begleitende Morbidität oder individuelle als auch prozessuale Risikofaktoren beeinflusst sein, denen nicht ausreichend Rechnung getragen wird. Hier wären Untersuchungen notwendig, bei denen gut charakterisierte Fälle der Spezialkliniken vergleichbaren Fällen der großen Notfallversorger gegenübergestellt werden.

Wie bereits oben erwähnt, stellen die drei hier untersuchten Krankheitsbilder nur einen Bruchteil typischer Notaufnahmediagnosen (Möckel et al. 2013 sowie Frick et al. 2017) dar. Die Vielzahl der dort gestellten Diagnosen macht deutlich, wie schwierig eine diagnosebezogene Qualitätssicherung in der Zentralen Notaufnahme ist und dass Variablen wie die primäre Symptomatik und Prozesszeiten stärker einbezogen werden müssen. Da die Notaufnahmen bisher keine eigene fachliche Zuordnung (fachliche Organisationseinheit) haben, beruhen alle Qualitätssicherungsmaßnahmen bisher auf fach- und oftmals diagnosebezogenen Maßnahmen. Diese berücksichtigen jedoch nicht, dass in der Notaufnahme diese Fälle erst aus einer großen Gruppe von Verdachtsfällen „herausgearbeitet“ werden. Insofern ist neben der Kennzeichnung des Ortes und der Eintreffzeit (siehe oben) auch die Bildung einer nationalen fachlichen Organisationseinheit „Notaufnahme“ überfällig.

\section{Wie können wir zukünftig die Notfallversorgung weiter verbessern und welche Projekte liefern hier Ansätze?}

Die anhaltende Debatte für eine Verbesserung der Notfallversorgung ist weiterhin vor allem von der Hypothese der Fehlsteuerung bestimmt. Crundsätzlich gilt, dass dabei zwei Arten der Fehlsteuerung unterschieden werden sollten, um Verbesserungsmöglichkeiten zu diskutieren: 
- Zum einen handelt es sich um diejenigen Patienten, deren Konstitution und Behandlungsdringlichkeit eine Versorgung im ambulanten Sektor erlauben würde. Diese kommen dort aber aus verschiedenen Gründen wie Öffnungszeiten, mangelnder Kommunikation oder Terminerhalt nicht an und suchen die Notaufnahmen auf (Stichwort Overcrowding). Studien zeigen dazu, dass bei ambulanten Vorstellungen in der Notaufnahme aus der Sicht der Betroffenen oftmals keine Alternative wahrgenommen wird, die aufzusuchen wäre (Schmiedhofer et al. 2016 sowie Oslislo et al. 2019).

- Zum anderen handelt es sich um die Patienten, die aus dringlichen Gründen notfallversorgt werden müssen, jedoch in dafür ungeeignete Krankenhäuser kommen. Mit Blick auf die Ergebnisse in diesem Beitrag kann aber festgehalten werden, dass ein homogener Standard in der Notfallversorgung zwar erstrebenswert ist, unabhängig davon die Patienten jedoch in vielen Fällen in Kliniken geleitet werden (oder diese aufsuchen), die nicht zur Behandlung ihres Krankheitsbildes geeignet sind.

So ist es wahrscheinlich, dass vor allem Patienten mit einer ungewissen Verdachtsdiagnose in Häusern versorgt werden, die nicht adäquat ausgestattet sind. Dass sie in diese Häuser gelangen, ist nicht allein ein Problem der Krankenhausstruktur, sondern insbesondere auch des Rettungsdienstes, der in weiten Teilen Deutschlands zu kleinteilig und interessensabhängig organisiert ist. Das laufende BMBF-geförderte Projekt EMANet beschäftigt sich intensiv u.a. mit der Struktur von Patientenzuweisungen und vulnerablen Cruppen in der Notaufnahme (Schmiedhofer et al. 2018).

Weitere Ansätze zur Verbesserung der Notfallversorgung im Krankenhaus wurden im Beitrag mehrfach erwähnt und beziehen sich wesentlich auf eine stärkere Berücksichtigung der Notaufnahme als eigenständigen fachlichen und strukturellen Leistungsbereich sowie eine bessere Datenerfassung.

Für einen so definierten Bereich liefert z.B. das Projekt ENQUIRE (http://www. enquire-projekt.de) relevante Daten: Durch die Identifikation von valide zu erfassenden und signifikant mit dem patientenrelevanten Outcome assoziierten Qualitätsindikatoren sollen Empfehlungen für die Verbesserung der Versorgung von Patienten in der Notaufnahme gegeben werden. Die prospektive Validierung der in vorbereitenden Arbeiten identifizierten Qualitätsindikatoren hinsichtlich ihrer Outcome-Relevanz ermöglicht die Risikoadjustierung derselben und unterstützt so die Entwicklung eines externen Benchmarkings für Notaufnahmen. Die Patientenversorgung kann dadurch optimiert und die Folgekosten nach einer Notfallbehandlung können gesenkt werden. Dabei sollte die diagnosebezogene Qualitätssicherung, die für die in diesem Beitrag untersuchten Bereiche sehr gut etabliert ist, um eine mehr prozessuale und diagnoseunabhängige Betrachtung ergänzt werden. 
Mit dem Datensatz Notaufnahme der Deutschen Interdisziplinären Vereinigung für Intensiv- und Notfallmedizin (DIVI) e.V. mit Unterstützung der Deutschen Gesellschaft für Interdisziplinäre Notfall- und Akutmedizin (DGINA) e.V. wurde ein deutschlandweiter Dokumentationsstandard für die Notaufnahmen geschaffen. Dieser ermöglicht nicht nur eine interdisziplinäre und interprofessionelle Informationsweitergabe, sondern stellt auch eine Basis für Qualitätssicherung und Benchmarking in den Notaufnahmen dar. Hier müssen zukünftig in den Kliniken Ressourcen geschaffen werden, die ein entsprechendes Qualitätsmanagement erlauben.

\section{Welchen Beitrag leisten dazu die Notfallstufen und wäre eine bundesweite Reform des Rettungsdienstes hilfreich?}

Die Notfallstufen haben einen wichtigen Beitrag geleistet, um den Ort und die strukturelle sowie in Teilen auch personelle Ausstattung der stationären Notfallversorgung festzulegen. Noch steht aus, ob die Stufeneinteilung an der Realität in der Notfallversorgung etwas ändern wird. Tatsächlich scheinen wesentlich stärker wirksame Faktoren, die ihre Ursache im Vergütungssystem haben, entscheidend. Der sogenannte „exit-block“ - also die Überfüllung der Notaufnahmen mit stationären Fällen, für die akut kein geeignetes Krankenhausbett zur Verfügung steht - sind das vorrangig zu bearbeitende Symptom (Möckel et al. 2013). Es bleibt abzuwarten, in welcher Form das anstehende Notfallversorgungsgesetz hier neue Impulse setzen kann. Jedenfalls könnte hier der Transportzwang für den Rettungsdienst entfallen und perspektivisch erstmals das Thema Fehlsteuerung effektiv adressiert werden. Vor dem Hintergrund der oben ausgeführten, vielfach subjektiven Alternativlosigkeit der Notaufnahmebehandlung bleibt als effektive Maßnahme nur die Strukturentwicklung der Notaufnahmen in Richtung von ausreichend finanzierten Kliniken für Notfallmedizin. Diese Strukturentwicklung wäre dann auch die Grundlage für ein effektives Qualitätsmanagement.

\section{Take home messages}

Nicht alle Kliniken, die Notfälle wie Herzinfarkte, Schlaganfälle und proximale Hüftfrakturen versorgen, sind personell und technisch adäquat dafür ausgestattet. Die Einführung von Notfallstufen ist nur ein bedingt wirkungsvolles Instrument.

- Die Vermutung, dass ein Zusammenhang zwischen den vereinbarten Notfallstufen und den Ergebnissen ausgewählter Qualitätsindikatoren bestünde, kann nicht eindeutig bestätigt werden. Bei einem nicht unerheblichen Anteil der Krankenhäuser und besonders bei denen der Basisnotfallversorgung (Stufe 1) korrespondiert die Notfallstufe sowohl quantitativ als auch qualitativ nicht mit der Versorgung von Notfällen. 
- Insbesondere spezialisierte Modulkliniken der Notfallstufe 0* weisen deutlich bessere Qualitätsergebnisse, wie zum Beispiel eine niedrigere 30-Tage-Sterblichkeit, auf. Die Mortalität liegt im Vergleich auch zu Krankenhäusern mit teilweise höheren Notfallstufen, an die analog höhere Anforderungen gestellt werden, unter dem Durchschnitt.

- Primäre Daten der Notfallversorgung stehen bisher in Routinedaten nur sehr eingeschränkt zur Verfügung, zumal die Lokalisation der Versorgung in einer Zentralen Notaufnahme nur indirekt erschlossen werden kann. Für die zukünftige Bewertung der Versorgungsqualität wäre ein Benchmark homogener Patientengruppen sinnvoll, in den Daten wie die primäre Symptomatik, Begleiterkrankungen, Vormedikation und Vitalparameter flächendeckend erhoben und einbezogen werden. Auch könnte die Einführung eines strukturierten Qualitätsmanagements im Hinblick auf die Qualität der Routinedatenerhebung in der Notaufnahme zu belastbareren Bewertungen des Versorgungsgeschehens beitragen.

- Die Einteilung der Krankenhäuser in Notfallstufen kann das Problem der strukturellen Fragmentierung, der kaum vorhandenen Vernetzung und der Intransparenz in der Notfallversorgung nur bedingt lösen. Zusätzlich scheinen die Notfallstufen nicht geeignet, um daraus eine indikationsbezogene Steuerung der Zuweisungen abzuleiten, da die Behandlungsqualität viel mehr von der spezialisierten Ausstattung des Hauses als von der eigentlichen Notfallstufe abhängt.

Eine optimierte Rettungskette, die insbesondere auf aktuell digital verfügbaren, zentral und transparent aufbereiteten Informationen beruht, würde wahrscheinlich zu weniger Verlegungen und einer geringeren Sterberate führen.

\section{Literatur}

Bolczek C, Nimptsch U, Möckel M, Mansky T (2019) Versorgungsstrukturen und Mengen-Ergebnisbeziehung beim akuten Herzinfarkt - Verlaufsbetrachtung der deutschlandweiten Krankenhausabrechnungsdaten von 2005 bis 2015. Gesundheitswesen D0I: 10.1055/a-0829-6580

Frick I, Möckel M, Müller R, Searle J, Somasundaram R, Slagman A (2017) Suitability of current definitions of ambulatory care sensitive conditions for research in emergency department patients: a secondary health data analysis. BMJ open 7: e016109

GKV-Spitzenverband (2018) Vereinbarung über Zu- und Abschläge für eine Teilnahme oder Nichtteilnahme von Krankenhäusern an der Notfallversorgung gemäß $\$ 9$ Absatz 1a Nummer 5 Klengt i.V.m. $\$ 136 c$ Absatz 4 SGB V (Notfallstufenvergütungsvereinbarung). URL: https://www.dkgev.de/fileadmin/default/Mediapool/2_Themen/2.3_Versorgung-Struktur/2.3.2_Gestuftes-System-Notfallstrukturen/Notfallstufenverguetungsvereinbarung.pdf (abgerufen am 19. Mai 2020)

G-BA (2013) Regelungen des Gemeinsamen Bundesausschusses gemäß $\oint 136 b$ Absatz 1 Satz 1 Nummer 3 SGB V über Inhalt, Umfang und Datenformat eines strukturierten Qualitätsberichts für nach $§ 108$ SGB V zugelassene Krankenhäuser (Regelungen zum Qualitätsbericht der Krankenhäuser, Qb-R). URL: https://www. dkgev.de/fileadmin/default/Mediapool/2_Themen/2.6._Qualitaet_Hygiene_und_Sicherheit/2.6.5._Qualitaetsbericht_und_Public_Reporting/Qb_R_2018-11-22_iK-2018-11-30.pdf (abgerufen am 10. Mai 2020) 
G-BA (2018) Regelungen des Gemeinsamen Bundesausschusses zu einem gestuften System von Notfallstrukturen in Krankenhäusern gemäß $\$ 136 c$ Absatz 4 des Fünften Buches Sozialgesetzbuch (SGB V). URL: https:// www.g-ba.de/downloads/62-492-2122/Not-Kra-R_2020-03-27_iK-2020-04-10.pdf (abgerufen am 19. Mai 2020)

Geissler A, Quentin W, Busse R (2017) Umgestaltung der Notfallversorgung: Internationale Erfahrungen und Potenziale für Deutschland. In: Krankenhaus-Report 2017: Schwerpunkt: Zukunft gestalten, Stuttgart: Schattauer, 41-59

Ibanez B, James S, Agewall S, Antunes MJ, Bucciarelli-Ducci C, Bueno H, Caforio ALP et al. (2018) ESC Guidelines for the management of acute myocardial infarction in patients presenting with ST-segment elevation: The Task Force for the management of acute myocardial infarction in patients presenting with ST-segment elevation of the European Society of Cardiology (ESC). Eur Heart I 39(2): 119-177

IQTIG (2018) Beschreibung der Qualitätsindikatoren für das Erfassungsjahr 2017: Hüftgelenknahe Femurfraktur mit osteosynthetischer Versorgung - Indikatoren 2017. URL: https://iqtig.org/downloads/auswertung/2017/17n1hftfrak/QSKH_17n1-HUEFTFRAK_2017_QIDB_V02_2018-04-25.pdf (abgerufen am 10. Mai 2020)

Knaak C, Brockhaus WR, Spies C et al. (2020) Presurgical cognitive impairment is associated with postoperative delirium and postoperative cognitive dysfunction. Minerva anestesiologica 86: 394-403

Kulla M, Brammen D, Greiner F et al. (2016) What do we need to assess the quality of care in German emergency departments? The development of a national data set, quality indicators and an emergency department registry by the DIVI. DIVI 7: 12-20

Mansky T, Drogan D, Nimptsch U, Günster C (2016) Eckdaten stationärer Versorgungsstrukturen für ausgewählte Krankheitsbilder in Deutschland. Qualitätsmonitor 2017. Hrsg. Dormann F und Klauber J. Berlin, Medizinisch Wissenschaftliche Verlagsgesellschaft

Möckel M, Searle J, Müller R et al. (2013) Chief complaints in medical emergencies: do they relate to underlying disease and outcome? The Charité Emergency Medicine Study (CHARITEM). European journal of emergency medicine: official journal of the European Society for Emergency Medicine 20: 103-8

Nimptsch U, Mansky T (2012) Trends in Acute Inpatient Stroke Care in Germany - an Observational Study Using Administrative Hospital Data From 2005-2010. Dtsch Arztebl Int 109: 885-92. DOI: 10.3238/arztebl.2012.0885.

Nimptsch U, Mansky T (2017) Hospital volume and mortality for 25 types of inpatient treatment in German hospitals: Observational study using complete national data from 2009 to 2014. BMJ Open 7:e016184. DOI: 10.1136/bmjopen-2017-01618

Nimptsch U, Mansky T (2020) G-IQI | German Inpatient Indicators Version 5.2. Bundesreferenzwerte für das Auswertungsjahr 2017. Working Papers in Health Services Research Vol. 2. Berlin: Universitätsverlag der Technischen Universität Berlin. DOI: http://dx.doi.org/10.14279/depositonce-10066

Oslislo S, Heintze C, Schmiedhofer M, Möckel M, Schenk L, Holzinger F (2019) How to decide adequately? Qualitative study of GPs' view on decision-making in self-referred and physician-referred emergency department consultations in Berlin, Germany. BMJ open 9: 0026786

Pross C, Busse R, Geissler A (2017) Hospital quality variation matters - A time-trend and cross-section analysis of outcomes in German hospitals from 2006 to 2014. Health Policy 121(8): 842-852. DOI: 10.1016/j.healthpol.2017.06.009

QSR-Klinikbericht (2019) QSR-Klinikbericht Verfahrensjahr 2019. Berichtsjahr 2015-2017 mit Nachbeobachtung 2018. Wissenschaftliches Institut der AOK (WId0). URL: https://www.qualitaetssicherung-mit-routinedaten.de/imperia/md/qsr/kliniken/klinikbericht_2019_muster_final.pdf (abgerufen am 19. Mai 2020)

Rapp K, Becker C, Todd C, Rothenbacher D, Schulz C, König HH, Liener U, Hartwig E, Büchele G (2020) Assoziation von orthogeriatrischem Co-Management und Sterblichkeit nach Hüftfraktur-Eine Beobachtungsstudie mit 58.000 Patienten aus 828 Krankenhäusern. Dtsch Arztebl Int 117: 53-9. DOI: 10.3238/arztebl.2020.0053

Rittberg W, Pflüger P, Ledwoch / et al. (2020) Zuweisungen von Notfallpatienten an abgemeldete Krankenhäuser (Akutbelegung). Dtsch Arztebl International 117: 465-71

Rosenthal M, Grunow II, Spies CD et al. (2020) Critical care guidelines on pain, agitation and delirium management: Which one to use? A systematic literature search and quality appraisal with AGREE II. Journal of critical care 59: $124-9$ 
Schmiedhofer M, Möckel M, Slagman A, Frick I, Ruhla S, Searle I (2016) Patient motives behind low-acuity visits to the emergency department in Germany: a qualitative study comparing urban and rural sites. BM] open 6: e013323

Schmiedhofer M, Inhoff T, Krobisch V et al. (2018) EMANet: A regional network for health services research in emergency and acute medicine. Zeitschrift fur Evidenz, Fortbildung und Qualität im Gesundheitswesen 135-136: 81-8

Somasundaram R, Geissler A, Leidel BA, Wrede CE (2018) Beweggründe für die Inanspruchnahme von Notaufnahmen - Ergebnisse einer Patientenbefragung. Das Gesundheitswesen 80(7):621-627. DOI: 10.1055/s0042-112459

Statistisches Bundesamt (2017) Fallpauschalenbezogene Krankenhausstatistik (DRG-Statistik) 2016. Fachserie 12, Reihe 6.4. Wiesbaden, Statistisches Bundesamt. URL: https://www.destatis.de/DE/Themen/Gesellschaft-Umwelt/Gesundheit/Krankenhaeuser/Publikationen/Downloads-Krankenhaeuser/fallpauschalenkrankenhaus-2120640167004.pdf?_blob=publicationFile (abgerufen am 19. Mai 2020)

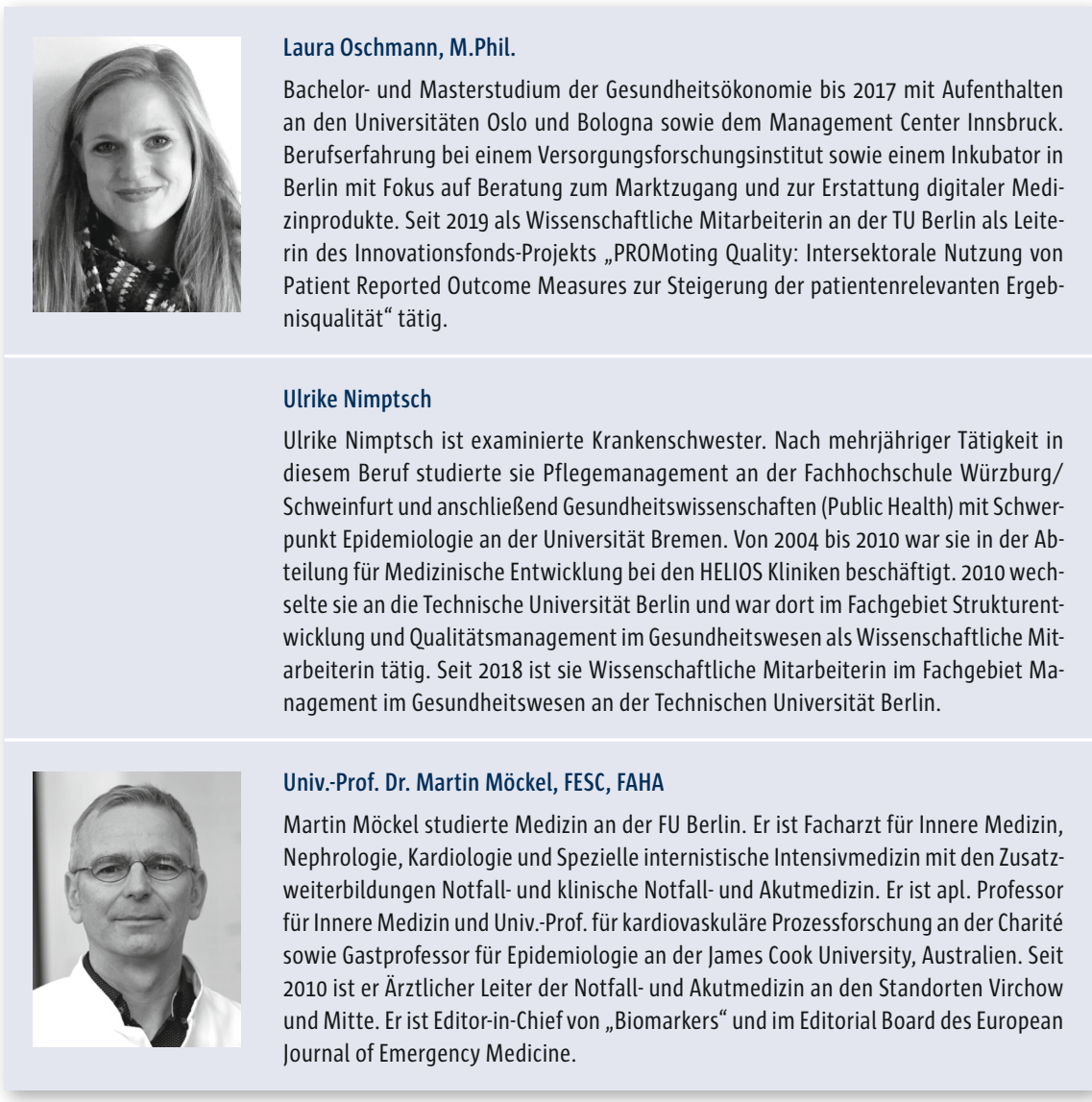




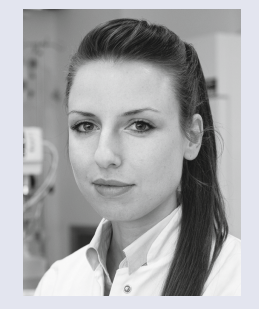

\section{Dr. Claudia Römer}

Claudia Römer studierte in Dresden Humanmedizin und absolvierte ihr Praktisches Jahr an der Queen Mary University of London und der Universität Zürich. Sie promovierte 2014 und ist seit 2015 klinisch in der Notfall- und Akutmedizin an der Charité bei Prof. Martin Möckel tätig und im Forschungsteam Notfall- und Akutmedizin wissenschaftlich aktiv. Berufsbegleitend absolvierte sie den Master of Public Health und ist Mitglied in den Fachgesellschaften DIVI und DGINA.

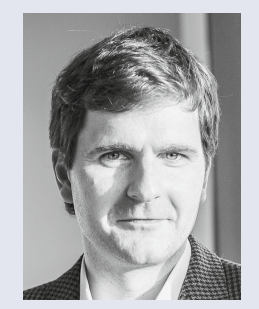

\section{Prof. Dr. Alexander Geissler}

Alexander Geissler ist promovierter Gesundheitsökonom und habilitierte sich für die Fächer Gesundheitssystemforschung und Gesundheitsökonomie an der TU Berlin. Seine Forschungsinteressen liegen in der Gesundheitsökonomie sowie Versorgungs- und Gesundheitssystemforschung. Er ist Inhaber des Lehrstuhls für Management im Gesundheitswesen und Direktionsmitglied der School of Medicine der Universität St. Gallen. 